ARTICLE

https://doi.org/10.1038/s41467-019-13875-y

\title{
MAP3Kinase-dependent SnRK2-kinase activation is required for abscisic acid signal transduction and rapid osmotic stress response
}

Yohei Takahashi(1) 1, Jingbo Zhang 1,3, Po-Kai Hsu (1) 1, Paulo H.O. Ceciliato', Li Zhang1, Guillaume Dubeaux (10 1, Shintaro Munemasa ${ }^{2}$, Chennan Ge ${ }^{1}$, Yunde Zhao (1) ${ }^{1}$, Felix Hauser (1) ${ }^{1}$ \& Julian I. Schroeder (1) ${ }^{1 *}$

Abiotic stresses, including drought and salinity, trigger a complex osmotic-stress and abscisic acid ( $A B A$ ) signal transduction network. The core ABA signalling components are snf1related protein kinase2s ( $\mathrm{SnRK} 2 \mathrm{~s}$ ), which are activated by $\mathrm{ABA}$-triggered inhibition of type2C protein-phosphatases (PP2Cs). SnRK2 kinases are also activated by a rapid, largely unknown, $\mathrm{ABA}$-independent osmotic-stress signalling pathway. Here, through a combination of a redundancy-circumventing genetic screen and biochemical analyses, we have identified functionally-redundant MAPKK-kinases (M3Ks) that are necessary for activation of SnRK2 kinases. These M3Ks phosphorylate a specific SnRK2/OST1 site, which is indispensable for ABA-induced reactivation of PP2C-dephosphorylated SnRK2 kinases. ABA-triggered SnRK2 activation, transcription factor phosphorylation and SLAC1 activation require these M3Ks in vitro and in plants. M3K triple knock-out plants show reduced ABA sensitivity and strongly impaired rapid osmotic-stress-induced SnRK2 activation. These findings demonstrate that this M3K clade is required for ABA- and osmotic-stress-activation of SnRK2 kinases, enabling robust $A B A$ and osmotic stress signal transduction.

\footnotetext{
${ }^{1}$ Division of Biological Sciences, Cell and Developmental Biology Section, University of California San Diego, La Jolla, CA 92093, USA. ${ }^{2}$ Graduate School of Environmental and Life Science, Okayama University, Tsushima-Naka, Okayama 700-8530, Japan. ${ }^{3}$ Present address: College of Resources and Environmental Sciences, National Academy of Agriculture Green Development, China Agricultural University, Beijing, PR China. *email: jischroeder@ucsd.edu
} 
imited water availability is one of the key factors that negatively impacts crop yields. The plant hormone abscisic acid (ABA) and the signal transduction network it activates, enhance plant drought tolerance through triggering of multiple cellular and developmental responses ${ }^{1-3}$. As plants are constantly exposed to changing water conditions, robustness of the $\mathrm{ABA}$ signal transduction cascade is important for plants to balance growth and drought stress resistance. Core ABA signalling components have been established ${ }^{2-7}$ : ABA receptors PYRABACTIN RESISTANCE (PYR/PYL)/REGULATORY COMPONENT OF ABA RECEPTOR (RCAR) inhibit type-2C proteinphosphatases (PP2Cs) ${ }^{4,5,8}$, resulting in activation of the snf1related protein kinase2 (SnRK2) protein kinases SnRK2.2, 2.3 and OST1/SnRK2.64,5,9-11. The SnRK2 kinases phosphorylate and thus regulate the activity of downstream components including transcription factors and ion channels $s^{9,10,12-15}$, which leads to changes in gene expression and stomatal closure. Activation of SnRK2 protein kinases requires phosphorylation of the SnRK2 kinases themselves, and in vitro experiments using purified recombinant OST1/SnRK2.6 suggest that phosphorylation of the activation-loop is an important step ${ }^{16}$. However, it has remained unclear whether direct autophosphorylation and/or transphosphorylation by unknown protein kinases reactivate these SnRK2 protein kinases in response to stress.

Previous studies showed that ABA-dependent phosphorylation of substrate proteins by SnRK2s could be reconstituted using only recombinant PYR/RCAR ABA receptors, PP2Cs, and SnRK2 proteins ${ }^{14,17,18}$. Recombinant SnRK2 proteins used in these studies, unlike SnRK2s in plant cells, have high intrinsic kinase activities even before ABA treatment ${ }^{16}$. Moreover, ABA receptors, SnRK2 kinases, PP2Cs and targets have generally been added to reactions simultaneously ${ }^{14,18}$. Therefore it is not clear whether autophosphorylation accounts for the ABA-dependent SnRK2 reactivation after $\mathrm{PP} 2 \mathrm{C}$-dependent inhibition in planta.

The Arabidopsis genome encodes ten SnRK2 kinases, and at least nine of these are activated in response to osmotic stress ${ }^{19}$. Interestingly, rapid osmotic stress-induced activation of SnRK2 protein kinases can occur independently of ABA signalling ${ }^{20}$. The osmotic stress sensing mechanisms and upstream signal transduction mechanisms leading to SnRK2 activation remain to a large degree unknown in plants.

In the present study, a family of MAP kinase kinase kinases (M3Ks) is identified that is essential for reactivation of SnRK2 protein kinases after PP2C dephosphorylation. We show that the OST1/SnRK2.6 protein kinase cannot reactivate itself after dephosphorylation. Three independent reconstitution assays and in planta analyses show the function of these M3Ks in SnRK2 kinase reactivation and $\mathrm{ABA}$ signalling. Moreover interestingly, triple $\mathrm{M} 3 \mathrm{~K}$ knockout mutant analyses show that the identified M3Ks are required for the rapid osmotic stress activation of SnRK2 kinases, in a less-well understood, previously proposed, pathway parallel to ABA signalling.

\section{Results}

Isolation of ABA-insensitive MAPKK-kinase amiRNA mutants. By unbiased forward genetic screening of seeds from over 1500 independent T2 artificial microRNA (amiRNA)expressing lines in pools ( 45,000 seeds screened) for ABAinsensitive seed germination, we isolated up to 290 putative mutants. In secondary screening of the surviving putative mutants in the next (T3) generation, progeny from 25 of the putative mutant plants continued to show a clearly reduced $\mathrm{ABA}$ sensitivity, including seeds propagated from three amiR-ax1117expressing plants (Fig. 1a-c). It is most likely that the three amiRNA-ax1117-expressing plants were the progeny of the same
amiRNA-expressing parent line. The amiR-ax1117 is predicted to target five subgroup B Raf-like MAPKK-kinase (M3Ks) genes (Supplementary Fig. 1). Previously, in a redundancycircumventing amiRNA pilot screen for impaired ABA inhibition of seed germination in Arabidopsis, we isolated putative mutants, including a $\mathrm{M} 3 \mathrm{~K}$ amiRNA-expressing line predicted to target seven MAPKK-kinases ${ }^{21}$. These seven putative target M3K genes overlap with four of the above amiR-ax1117 target genes (Supplementary Fig. 1). Furthermore, in additional genetic screens for ABA-insensitive inhibition of seed germination using more than 2,000 pooled amiRNA-expressing lines ( $~ 50,000$ seeds screened), we again isolated the previously isolated $m 3 k$ amiRNA line two more times. The amiR-ax1117 amiRNA and the $m 3 k$ amiRNA target five and seven overlapping Arabidopsis Raf-like kinase members from subgroup B1 and B3 (Supplementary Fig. 1). Note that the Arabidopsis genome includes $\sim 80 \mathrm{M} 3 \mathrm{~K}$ genes and $22 \mathrm{~B}$ family M3K members ${ }^{22}$. Because SnRK2 protein kinase activation is a key step in ABA signalling, and based on prior findings described further below (Fig. 1f), we investigated ABA-activation of SnRK2 protein kinase activity in seedlings of the $m 3 k$ amiRNA line by in-gel kinase assays. SnRK2 protein kinases are detected at apparent mobilities of $40-44 \mathrm{kDa}$ in in-gel kinase assays ${ }^{10,23}$. Interestingly, ABA-activation of kinase activities was reduced by $60 \%$ in the $m 3 k$ amiRNA line (Fig. 1d, e, Supplementary Fig. 2; $n=3$ experiments).

OST1/SnRK2.6 reactivation after dephosphorylation. We investigated phosphorylation of purified recombinant GSTtagged OST1/SnRK2.6 protein kinase after dephosphorylation in vitro. To test whether OST1/SnRK2.6 could be re-activated by autophosphorylation, after dephosphorylation, the GST-OST1/ SnRK2.6 protein bound on glutathione sepharose 4B resin was incubated with calf intestinal alkaline phosphatase (CIAP), and $\left[\gamma^{32} \mathrm{P}\right]-\mathrm{ATP}$ was added to the reaction after wash out of CIAP. Surprisingly, we found that OST1/SnRK2.6 showed very low autophosphorylation activity even after the protein phosphatase had been removed (Fig. 1f, lane $2 ; n=3$ experiments). Other ABA signalling protein kinases including the calcium-dependent protein kinases $\mathrm{CPK}^{24}$ and $\mathrm{CPK} 23^{25}$ and the MAP kinase MPK12 26,27 did not phosphorylate OST1/SnRK2.6 after dephosphorylation (Fig. 1f). Interestingly these results implied that autophosphorylation is not sufficient for OST1/SnRK2.6 reactivation following protein phosphatase exposure and removal. Therefore, another unknown protein kinase may be required for reversible ABA signal transduction.

We investigated whether the amiRNA-targeted M3Ks may directly activate OST1/SnRK2.6. In-gel kinase assays were carried out in vitro after incubation of the dephosphorylated His-OST1/ SnRK2.6 with GST-tagged recombinant M3K kinase domains and His-tagged full-length M3Ks in the presence of ATP. Notably, three M3Ks from the subgroup $\mathrm{B} 3$, named $\mathrm{M} 3 \mathrm{~K} \delta 1, \delta 6$, and $\delta 7$, were found to strongly activate OST1/SnRK2.6, whereas the other M3Ks targeted by the corresponding amiRNA did not clearly activate OST1/SnRK2.6 under the imposed conditions in vitro (Fig. $1 \mathrm{~g}$ and Supplementary Fig. $3 ; n=3$ experiments). OST1/ SnRK2 kinase activation was not induced by an inactive mutant M3K kinase protein, M3K 66 (K775W) (Fig. 2a). Moreover, the $\mathrm{M} 3 \mathrm{~K} \delta 1, \delta 6$, and $\delta 7$ kinase domains directly phosphorylated the kinase inactive OST1/SnRK2.6 (D140A) mutant isoform (Fig. 2b and Supplementary Fig. 4). Note that a Physcomitrella patens protein kinase ARK showing similarity to these M3Ks was recently reported to phosphorylate a Physcomitrella SnRK2 kinase ${ }^{28}$.

M3Kס1 phosphorylates a critical Ser171 for OST1activation. Mass spectrometry analyses revealed that $\mathrm{M} 3 \mathrm{~K} \delta 1$ phosphorylated 
a

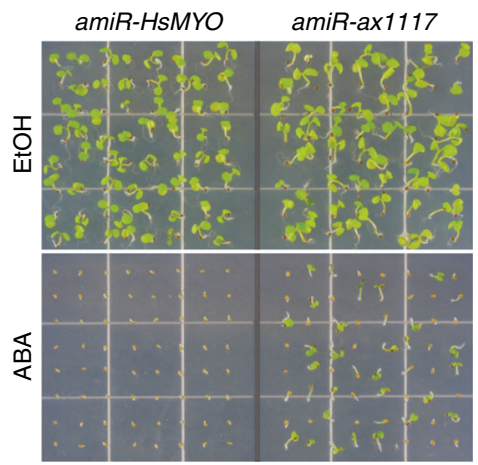

b

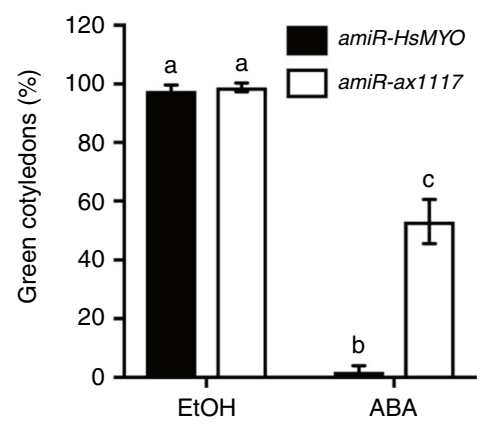

C

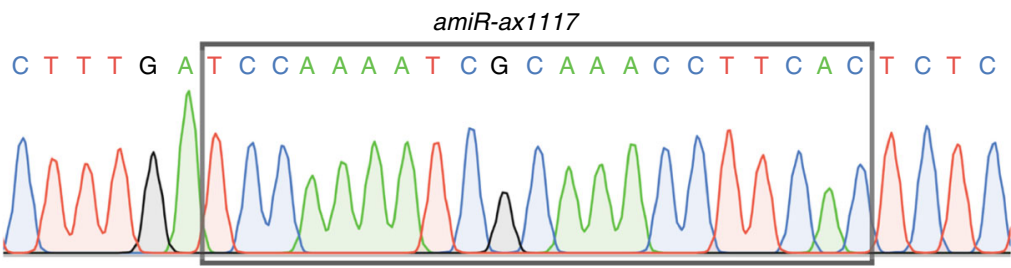

d

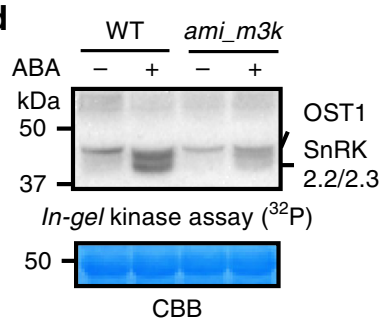

e

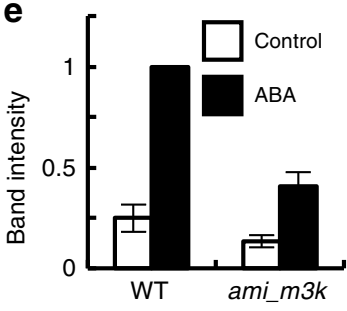

f

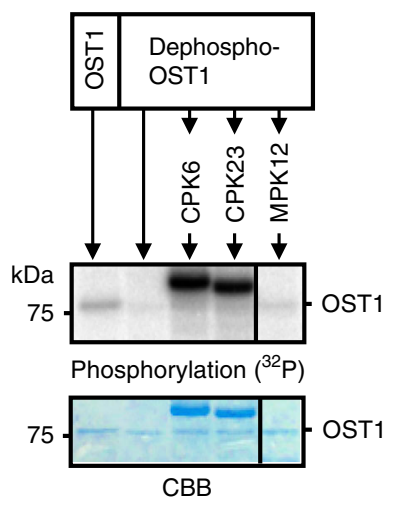

g

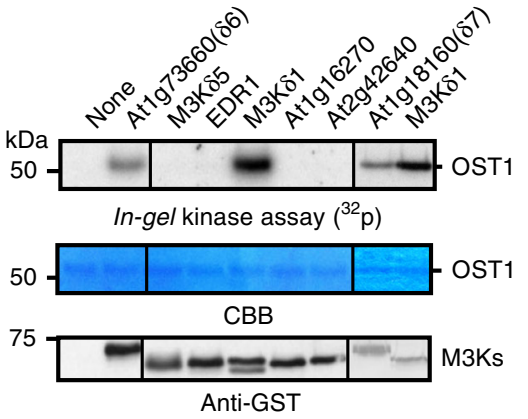

Fig. 1 Identification of MAPKK-kinases that reactivate OST1/SnRK2 kinases by phosphorylation. a Seeds of amiR-HsMYO wild-type (control line) or amiR-ax1117 mutant were sowed on 1/2 MS medium containing $2 \mu \mathrm{M} \mathrm{ABA}$, or $0.02 \% \mathrm{EtOH}$ as control, for germination assays. Representative images showing seed germination after 6 days. $\mathbf{b}$ The percentage of seedlings showing green cotyledons was analyzed. Data represent mean \pm s.d. $n=4$ experiments. Each experiment included 64 seeds for each genotype. Letters at the top of columns are grouped based on two-way ANOVA and Tukey's test, $P<0.05$. $\mathbf{c}$ Identification of the amiRNA sequence in amiR-ax1117 plants. Black box labels the sequence of amiR-ax1117. The amiR-ax1117 is predicted to

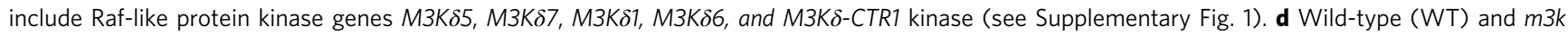
amiRNA seedlings were incubated with $10 \mu \mathrm{M}$ ABA for $15 \mathrm{~min}$. In-gel kinase assays were performed using histone type III-S as a substrate. e SnRK2 band intensities as shown in $\mathbf{d}$ were measured using Image,$n=3$ experiments, error bars show \pm s.e.m. $\mathbf{f}$, Recombinant GST-OST1/SnRK2.6 protein was dephosphorylated by alkaline phosphatase in vitro and used for in vitro phosphorylation assays after incubation with CPK6, CPK23 or MPK12 protein kinases. Note visible autophosphorylation activity of CPK6 and CPK23. $\mathbf{g}$ Dephosphorylated recombinant His-OST1/SnRK2.6 protein was incubated with kinase domains of seven M3Ks and used for in-gel kinase assays (phylogenetic tree: see Supplementary Fig. 1). Note lanes on the left are from the same gel as lanes in the middle section.

the OST1/SnRK2.6 residues Ser171, Ser175, and Thr176 in the OST1-activation loop (Fig. 2c). We next focused on Ser171, because this site has not been found as an OST1/SnRK2.6 autophosphorylation site in vitro ${ }^{16}$, consistent with our mass spectrometry analyses of OST1 (Fig. 2c, d). Using Arabidopsis mesophyll cell protoplasts as a transient expression system, consistent with a previous study ${ }^{11}$, we found that substitution of this OST1/SnRK2.6 Ser171 by an alanine completely abrogated ABA-dependent activation of OST1/SnRK2.6 (Fig. 2e; $n=3$ experiments).
Notably, the OST1-S171A mutation does not disrupt kinase activity in vitro, while another phosphorylation site mutation (S175A) disrupts kinase activity (Supplementary Fig. 5a, b). An OST1/SnRK2.6 T176A mutation does not disrupt kinase activity in vitro nor does the T176A mutation affect ABA activation of OST1/SnRK2.6 in vivo (Supplementary Fig. 5a, b). These results suggest that Ser171 plays an important role in ABA-activation of OST1/SnRK2.6 in plant cells. A potential phospho-mimic isoform of Ser171, OST1/SnRK2.6 (S171E) has no detectable kinase activity in mesophyll cells (Supplementary Fig. 5c). This is 
a

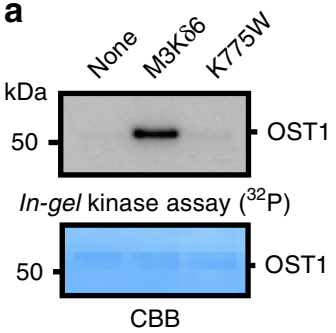

b

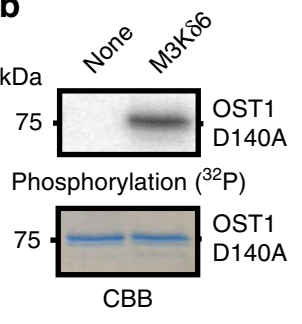

c

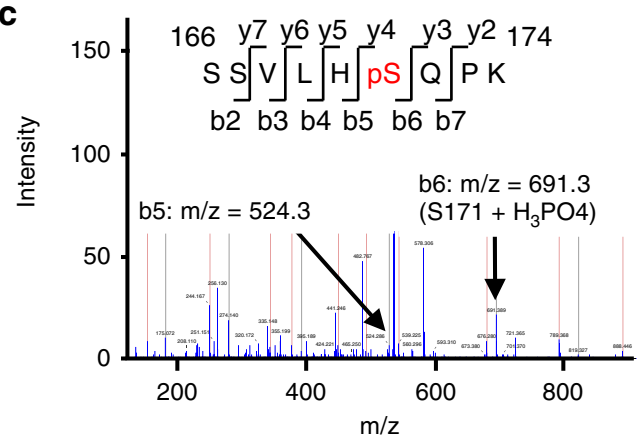

d

\begin{tabular}{|c|c|}
\hline OST1 only & ${ }^{171} S Q P K \underline{S}^{175}$ \\
\hline$+\mathrm{M} 3 \mathrm{~K} \delta 1$ & ${ }^{171} \underline{S} Q P K \underline{S}^{175}$ \\
\hline
\end{tabular}

e

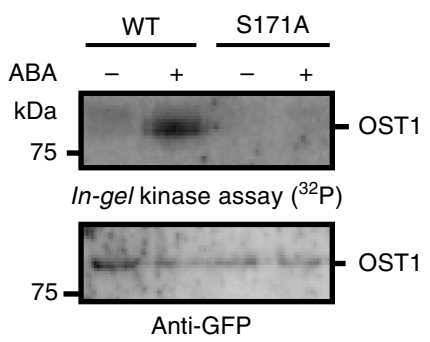

Fig. 2 MAPKK-kinase-induced OST1/SnRK2.6 Ser171 phosphorylation is essential for ABA activation of OST1/SnRK2.6 activation. a The inactive M3K $\delta 6$ kinase domain mutant (K775W) did not reactivate His-OST1/ SnRK2.6 in vitro. b Inactive GST-OST1/SnRK2.6-D140A kinase protein was incubated with M3K $\delta 6$ kinase domain, and in vitro phosphorylation assays were performed with ${ }^{32} \mathrm{P}$-ATP. c Recombinant inactive OST1(D140A) and $M 3 K \delta 1$ kinase domains were incubated with ATP. A mass spectrum of phosphorylated OST1 peptide (SSVLHpSQPK) is shown. pS indicates phosphorylated Ser171 of OST1(D140A). d Phosphorylation at Ser171 was not detectable after in vitro autophosphorylation of OST1/SnRK2.6, but was consistently phosphorylated in the presence of M3K $\delta 1$. e OST1(S171A)-GFP was transiently expressed in Arabidopsis mesophyll cell protoplasts. Protoplasts were incubated with $10 \mu \mathrm{M}$ ABA or control buffer for 15 min, and OST1/SnRK2.6 activities were analyzed by in-gel kinase assays.

consistent with a previously reported OST1/SnRK2.6 (S171D) mutant protein $^{11}$. We further investigated the effect of ABA on phosphorylation of OST1-S171 in mesophyll cells. Ser171 is phosphorylated in plant mesophyll cells in response to ABA (Supplementary Fig. 6) ${ }^{10,11}$.

We created transgenic Arabidopsis plants stably expressing OST1-HF (S171A) in the ost1-3 background ${ }^{29,30}$. Expression of OST1-HF (S171A) did not rescue the ABA-insensitive stomatal conductance response and the low leaf temperature phenotype of the ost1-3 mutant in two independent lines (Fig. 3a-c and Supplementary Fig. 7). Complementation of ost1-3 with the wildtype OST1-HF isoform restored ABA-induced stomatal closing and warm leaf temperatures (Fig. 3a-c and Supplementary Fig. 7), together indicating that Ser171 is required for OST1/SnRK2.6 function in stomatal closing (Fig. 3a-c and Supplementary Fig. 7).

Patch-clamp analyses of the ost1-3 complementation lines showed the essential role of Ser171 for ABA-induced S-type anion channel activation in Arabidopsis guard cells (Fig. 3d, e). We further found that, in contrast to OST1-HF-expressing controls, OST1-HF (S171A) was not activated in Arabidopsis mesophyll cells in response to ABA in these stable homozygous transgenic plant lines (Fig. 3f).

Reconstitution of early ABA signalling with MAPKK-kinases. Previous studies have reconstituted ABA-dependent phosphorylation of OST1/SnRK2.6 substrates in vitro using recombinant proteins ${ }^{14,18}$. Recombinant OST1/SnRK2.6 has many phosphorylated sites and a significant protein kinase activity in vitro ${ }^{16}$. However, we find that prior dephosphorylated OST1/SnRK2.6 could unexpectedly not be re-activated by itself (Fig. 1f). We therefore hypothesized that these $\mathrm{M} 3 \mathrm{Ks}$ have a role in reactivation of SnRK2 after inactivation by PP2C-mediated dephosphorylation. To test this, we pursued in vitro reconstitution experiments using recombinant proteins PYR1/RCAR11, the HAB1 PP2C, OST1/ SnRK2.6 with or without M3Kס6. In-gel kinase assays clearly showed that when HAB1-dependent OST1/SnRK2.6 dephosphorylation preceded ABA application, PYR1/RCAR11, HAB1, and OST1/SnRK2.6 could not recover OST1/SnRK2.6 activation (Fig. $4 \mathrm{a} ; n>3$ experiments). Moreover, OST1/SnRK2.6 was no longer activated even after ABA treatment (Fig. $4 \mathrm{a} ; n>3$ experiments). However, the OST1/SnRK2.6 kinase was clearly reactivated in response to ABA when M3K $\delta 6$ was added to these reactions (Fig. $4 \mathrm{~b} ; n>3$ experiments). Consistent with these findings, in vitro reconstitution of ABA-dependent AKS1 transcription factor phosphorylation by OST1/SnRK2. $6^{18}$ was not observed when ABA was added after OST1/SnRK2.6 had been initially dephosphorylated by the PP2C HAB1 for 10 min (Fig. 4c, compare lanes $5,6)$. Addition of M3K $\delta 6$ restored ABA-induced His-AKS1 phosphorylation (Fig. 4c, compare lanes 7,8 ).

Reconstitution of ABA activation of SLAC1 requires M3Ks. OST1/SnRK2.6-mediates activation of the S-type anion channel SLAC1 in Xenopus oocytes ${ }^{12,13}$, and ABA-induced SLAC1 activation was reconstituted in oocytes ${ }^{17}$. These results strongly depended on artificial BiFC tags that force interaction of the SLAC1 channel with OST1/SnRK2.6 proteins $^{12,17}$, indicating that the BiFC tag might cause an unknown artificial effect. When expressing SLAC1 and OST1/SnRK2.6 proteins without any tag in oocyte experiments in the present study, SLAC1 was not significantly activated (Supplementary Fig. 8a-e). We found that SLAC1 was strongly activated when small amounts of $M 3 K \delta 1$, $M 3 K \delta 6$, or $M 3 K \delta 7$ cRNA were co-injected with OST1 into oocytes (Supplementary Fig. 8a-e; ratio of [M3K] to [OST1] cRNA $=1$ to 10$)$. However, the M3Ks did not activate SLAC1 in the absence of OST1/SnRK2.6 (Supplementary Fig. 8a-e), even when the injected M3K to SLAC1 cRNA concentration ratio was 1 to 1 . Furthermore, kinase inactive OST1/SnRK2.6 (D140A) does not activate SLAC1 in the presence of M3K $\delta 1$ (Supplementary Fig. 8f, g).

In additional experiments, we co-injected cRNA for the ABA receptor PYL9/RCAR1, together with the ABI1 PP2C, OST1/ SnRK2.6, SLAC1, and M3Ks into oocytes, to test whether ABAdependent SLAC1 anion channel activation could be reconstituted with these components. ABA could activate SLAC1 in oocytes only in the presence of low concentrations of either $M 3 K \delta 1, M 3 K \delta 6$ or $M 3 K \delta 7$ mRNAs (Fig. 4d-f). Moreover, inactive $\mathrm{M} 3 \mathrm{~K}$ kinase mutant isoforms and inactive OST1 
a

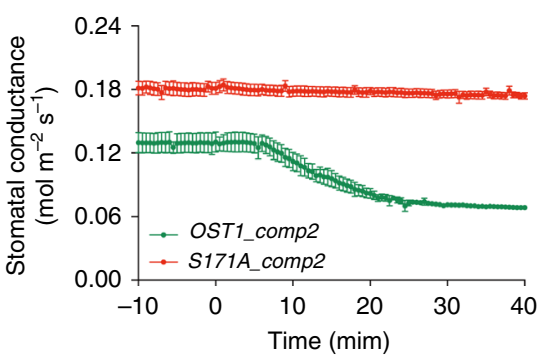

d

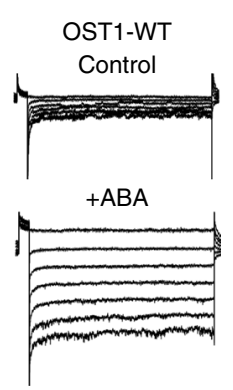

b

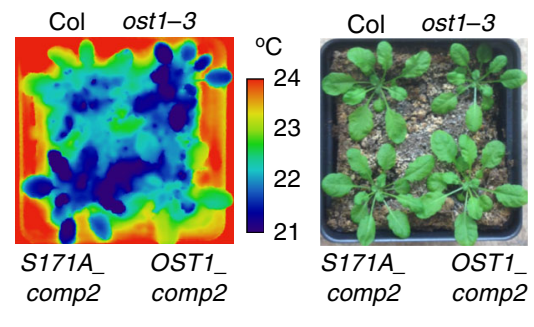

C

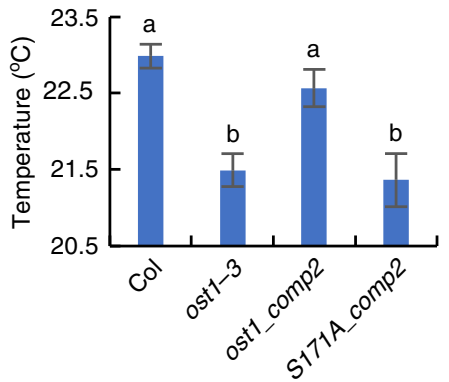

e

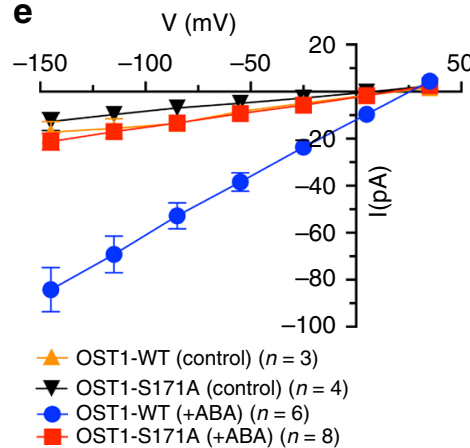

f

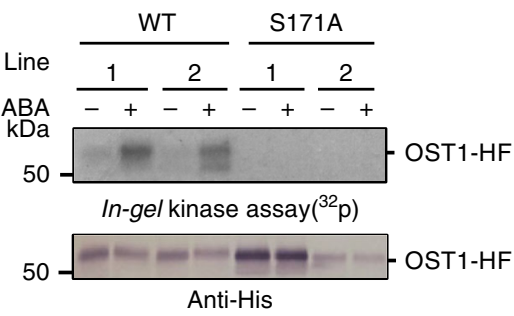

Fig. 3 OST1/SnRK2.6 Ser171 is essential for ABA-induced stomatal closure and S-type anion channel activation in planta. a Stomatal conductances were analyzed in intact detached leaves of stable transgenic Arabidopsis [pUBQ10:OST1-HF/ost1-3 (OST1-comp2) and pUBQ10:OST1-S171A-HF/ost1-3 (S171Acomp2)]. $2 \mu \mathrm{M}$ ABA was applied to petioles at 0 min. Data presented are means \pm s.e.m. $(n=4$ leaves from four plants for each genotype). $\mathbf{b}$ Leaf temperatures of Col, ost1-3, OST1-comp2 and S171A-comp2 were measured by thermal imaging. Plants were sprayed with $20 \mu \mathrm{M}$ ABA, and thermal images were taken after $3 \mathrm{~h}$. The bright field image shows where leaves from neighboring plants overlapped. c, Leaf temperatures were measured by using Fiji software ( $n=5$ experiments, means \pm s.e.m.). Letters at the top of columns are grouped based on one-way ANOVA and Tukey's test, $P<0.05$. $\mathbf{d}$ ABAactivated S-type anion channel currents were investigated by patch-clamp analyses using guard cell protoplasts from the transgenic Arabidopsis lines pUBQ10:OST1-HF/ost1-3 (OST1-WT) and pUBQ10:OST1-S171A-HF/ost1-3 (OST1-S171A). e Average current-voltage relationship of S-type anion channel as shown in d. Data presented are means \pm s.e.m. $\mathbf{f}$ Kinase activities of OST1(S171A) in mesophyll cells from stably-transformed homozygous transgenic plants were investigated by in-gel kinase assays. Protoplasts were incubated with $10 \mu \mathrm{M}$ ABA for 15 min.

(S171A) disrupted reconstitution of SLAC1 activation (Supplementary Fig. 9). As SLAC1 plays an important role in ABAinduced stomatal closing, gas exchange experiments were pursued. $m 3 k$ amiRNA plants show a reduced steady-state stomatal conductance and an $\mathrm{ABA}$ insensitivity in stomatal closure (Supplementary Fig. 10a, b).

The reduced steady-state stomatal conductance in the $m 3 k$ amiRNA line indicates additional effects of this artificial microRNA and/or compensatory effects of impaired stomatal closing response mutants ${ }^{31,32}$. Higher order mutant combinations will be required to investigate this hypothesis. Based on the lower steady-state stomatal conductance, the impaired response to $\mathrm{ABA}$ (Supplementary Fig. 10a, b) and findings showing that ABA activation of S-type anion channels is an important mechanism for ABA-induced stomatal closing 24,33 , we investigated ABA activation of S-type anion channels in guard cells. ABA $(10 \mu \mathrm{M})$ caused typical ABA activation of S-type anion currents in guard cells of the wild-type (Col-0) and the HsMYO control line (Supplementary Fig. 10c-f). In contrast, ABA activation of S-type anion channels was impaired in guard cells of the $m 3 k$ amiRNA line (Supplementary Fig. 10g, h). ABA signalling reconstitution (Fig. 4) and guard cell anion channel regulation analyses (Supplementary Fig. 10c-h) together suggest that the identified M3Ks provide a missing component of the early ABA signalling module.

Higher order M3K mutants show ABA-insensitive phenotypes. We isolated T-DNA insertion mutants [ $m 3 k \delta 1$ (SALK_048985), $m 3 k \delta 6-1$ (SALK_004541), $m 3 k \delta 6-2$ (SALK_001982), and $m 3 k \delta 7$
(SALK_082710)] (Fig. 5a). We also deleted large fragments of the $M 3 K \delta 1$ or $M 3 K \delta 7$ genes by CRISPR-Cas9 in the $m 3 k \delta 6-2$ T-DNA knockout background (Fig. 5b), and a triple knockout mutant ( $m 3 k \delta 1$ crispr $m 3 k \delta 6-2 m 3 k \delta 7 c r i s p r)$ was generated by crossing these lines (Fig. $5 c$ ) to analyze the physiological functions of these M3K genes. The $m 3 k \delta 1$ crispr $m 3 k \delta 6-2 m 3 k \delta 7 c r i s p r$ triple mutant showed a reduced ABA sensitivity phenotype in green cotyledon emergence from seeds (Fig. $5 \mathrm{~d}$, e). The double mutants $m 3 k \delta 1$ $m 3 k \delta 7$ and $m 3 k \delta 6-2 m 3 k \delta 7$ showed weaker ABA-insensitive phenotypes than the triple mutants (Supplementary Fig. 11a, b). Also, $m 3 k \delta 1 / \delta 6-1 / \delta 7$ mutant seedlings showed a reduced ABA sensitivity in inhibition of primary root elongation on $1 / 2 \mathrm{MS}$ plates supplemented with ABA (Supplementary Fig. 11c).

We confirmed knockout of full-length expression of $M 3 K \delta 1$ and $M 3 K \delta 7$ in the T-DNA lines, while there was partial expression of the kinase domain of $M 3 K \delta 6$ in the $m 3 k \delta 6-1$ line (Fig. 5f). Seed germination analyses showed reduced ABA sensitivity in the $m 3 k \delta 1 m 3 k \delta 6-1 m 3 k \delta 7$ T-DNA insertion triple mutants (Fig. 5g, h). Another T-DNA allele for $M 3 K \delta 6$ for which the full length and kinase domain transcripts could not be amplified (Fig. 5a; $m 3 k \delta 6-2$ ) was considered. However, we could not isolate a viable $m 3 k \delta 1 m 3 k \delta 6-2 m 3 k \delta 7$ triple mutant, possibly due to homozygous lethality, likely linked to an unknown second site mutation. Because the partial expression of the $M 3 K \delta 6$ kinase domain fragment was detected in the $m 3 k \delta 6-1$ mutant (Fig. 5f), this kinase fragment may weaken the phenotypic effect.

To further test the function of these M3Ks, we created amiRNA lines predicted to target only the triple combination of $M 3 K \delta 1$, $M 3 K \delta 6$, and $M 3 K \delta 7$ and found that three independent amiRNA lines showed ABA-insensitivities in seed germination (Fig. 5i, j). 
a

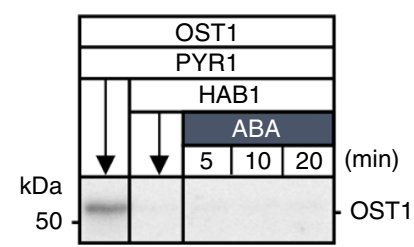

b

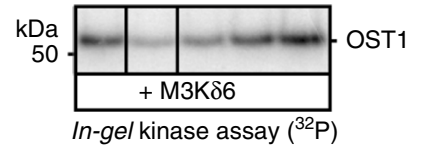

c

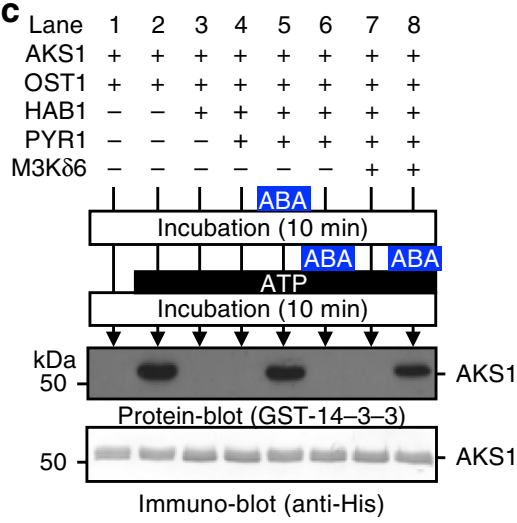

d

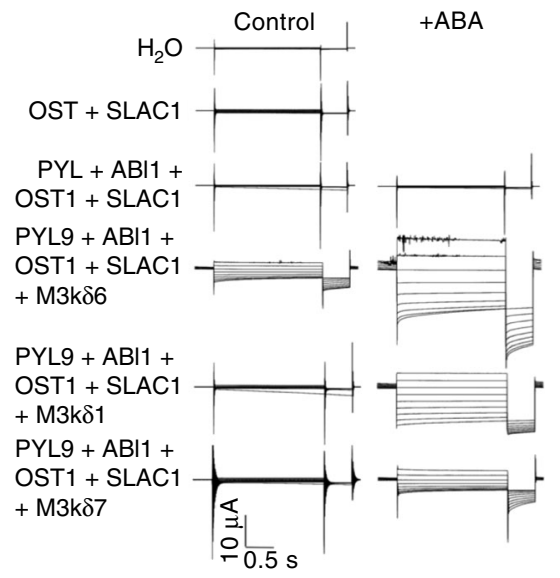

e

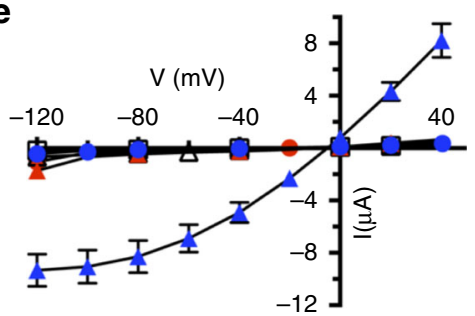

Ð $\mathrm{H}_{2} \mathrm{O}(N=12) \quad \triangle \mathrm{OST} 1+\operatorname{SLAC1}(N=10)$

- PYL9 + ABI1 + OST1 + SLAC1 $(N=8)$

- $\mathrm{PYL} 9+\mathrm{ABI} 1+\mathrm{OST} 1+\mathrm{SLAC} 1+\mathrm{ABA}(N=7)$

$\pm \mathrm{PYL} 9+\mathrm{AB} 1+\mathrm{OST} 1+\mathrm{SLAC1}+\mathrm{M} 3 \mathrm{~K} \delta 1(N=10)$

$\pm \mathrm{PYL} 9+\mathrm{ABI} 1+\mathrm{OST} 1+\mathrm{SLAC} 1+\mathrm{M} 3 \mathrm{~K} \delta 1+\mathrm{ABA}(N=8)$

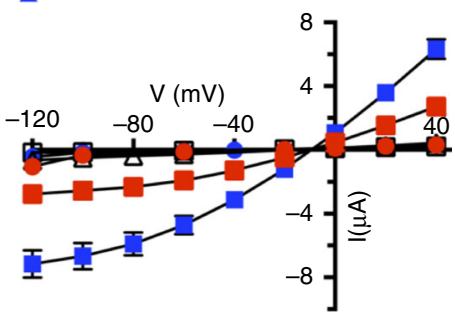

$\square \mathrm{H}_{2} \mathrm{O}(N=12) \quad \triangle \mathrm{OST} 1+\operatorname{SLAC1}(N=10)$

- PYL9 + ABI1 + OST1 + SLAC1 $(N=8)$

- $\mathrm{PYL} 9+\mathrm{ABI} 1+\mathrm{OST} 1+\mathrm{SLAC} 1+\mathrm{ABA}(N=7)$

를 PY $9+\mathrm{ABI} 1+\mathrm{OST} 1+\mathrm{SLAC} 1+\mathrm{M} 3 \mathrm{~K} \delta 6(N=11)$

- PYL9 + ABI1 + OST1 + SLAC1 + M3K $66+\mathrm{ABA}(N=14)$

f

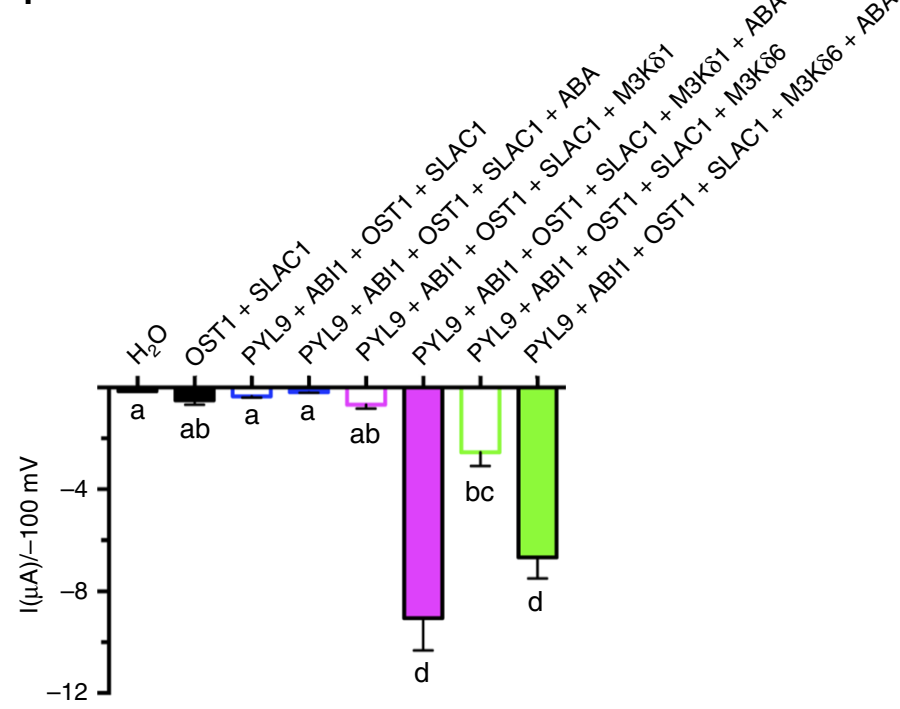

Fig. 4 MAPKK-kinases are essential for ABA signalling module. $\mathbf{a}$, b In vitro reconstitution of ABA-induced OST1/SnRK2 activation without M3K $\delta 6$ (a) or with M3K $\delta 6$ (b). The recombinant proteins His-PYR1/RCAR11, His-OST1/SnRK2.6 without (a) or with (b) GST-M3K $\delta 6$ kinase domain were mixed. After addition of His-HAB1, protein solutions were incubated for $10 \mathrm{~min}$. Then, $50 \mu \mathrm{M}$ ABA was added to the protein solution. Reactions were stopped at the indicated times. OST1/SnRK2.6 kinase activities were detected by in-gel kinase assays. c Recombinant His-PYR1/RCAR11, His-HAB1, His-OST1/SnRK2.6, His-AKS1, and GST-M3K $\delta 6$ kinase domain were mixed as indicated above the gel. $50 \mu$ M ABA was added before (lane 5) or after (lanes 6 and 8 ) 10 min incubation at room temperature. Then, $100 \mu \mathrm{M}$ ATP was added (lanes 2-8) to trigger phosphorylation reactions for 10 min. Note that M3K $\delta 6$ is required for ABA-induced AKS1 phosphorylation when ABA is added $10 \mathrm{~min}$ after exposure to HAB1-PP2C-including mix (compare lanes 6 and 8 ). Reactions were stopped by addition of SDS-PAGE loading buffer. Phosphorylation of AKS1 is detected by binding of 14-3-3Phi (At1g35160) to the phosphorylated AKS1 protein ${ }^{15}$. AKS1 phosphorylation is shown by protein-blot (top), and protein amount is monitored by immuno-blot (bottom). d-f Reconstitution of ABAactivation of SLAC1 channels in Xenopus oocytes, in the presence or absence of M3Ks. $\mathbf{d}$ Representative whole-cell chloride current recordings of oocytes co-expressing the indicated proteins, without (control) or with injection of $50 \mu \mathrm{M} \mathrm{ABA}(+\mathrm{ABA})$. Currents were recorded in response to voltage pulses ranging from $+40 \mathrm{mV}$ to $-120 \mathrm{mV}$ in $-20 \mathrm{mV}$ steps with a holding potential at $0 \mathrm{mV}$ and a final tail potential of $-120 \mathrm{mV}$. e Mean current-voltage curves of oocytes co-expressing the indicated proteins, with or without injection of $A B A$. The symbols of $\mathrm{H}_{2} \mathrm{O}$ control, OST1 + SLAC1, PYL9/RCAR1 + ABI1 + OST1 + SLAC1, PYL9/RCAR1 + ABI1 + OST1 + SLAC1 + ABA, and PYL9/RCAR1 + ABI1 + OST1 + SLAC1 + M3Ks overlapped. Single symbols are shown for some data points for better viewing. $\mathbf{f}$ Average SLAC1-mediated currents at $-100 \mathrm{mV}$, co-expressing the indicated proteins, in the presence or absence of $50 \mu \mathrm{M}$ ABA. Data from three independent batches of oocytes showed similar results. One representative batch of oocytes is shown, with the number of oocytes in that batch indicated in parentheses. $\mathrm{H}_{2} \mathrm{O}$, OST1 + SLAC1, PYL9/RCAR1 + ABI1 + OST1 + SLAC1, and PYL9/RCAR1 + ABI1 + OST1 + SLAC1 + $A B A$ controls are the same data in both panels in $\mathbf{e}$ as the data are from the same oocyte batch. Error bars denote mean \pm s.e.m. Means with letters (a, $\mathbf{b}, \mathbf{c}$, and d) are grouped based on one-way ANOVA and Tukey's multiple comparisons test, $P<0.05$. 
a

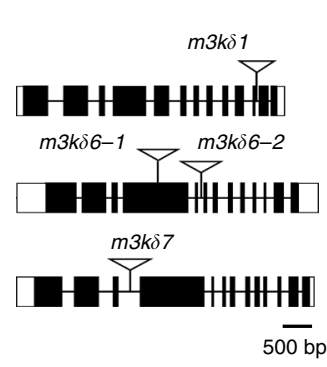

b
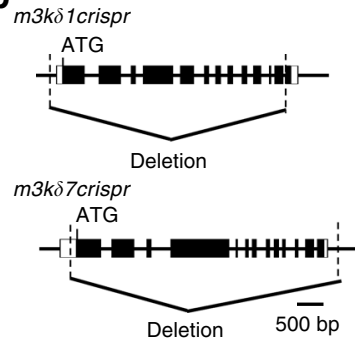

C

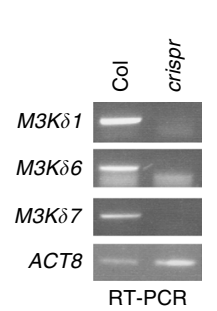

d

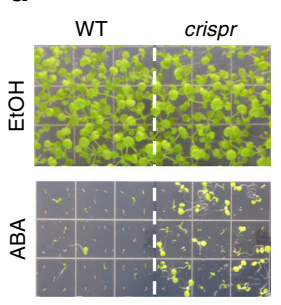

e ETOH $\square$ ABA

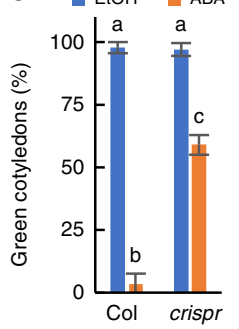

$\mathbf{f}$

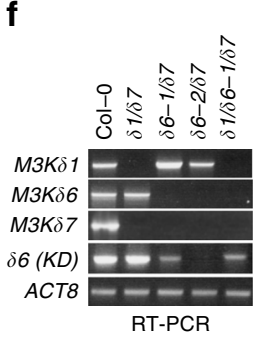

g

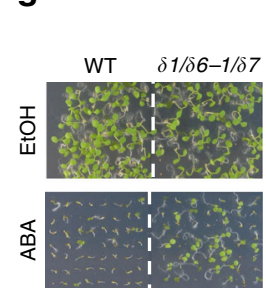

h

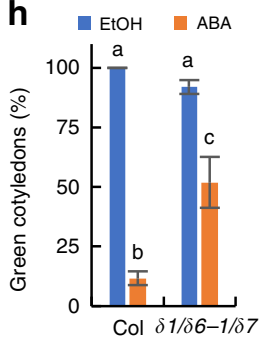

i
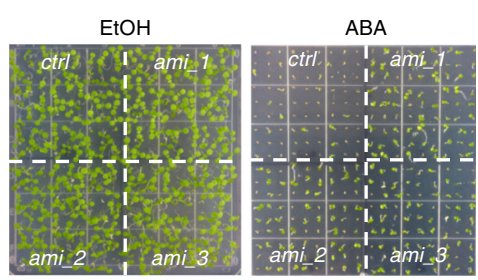

j

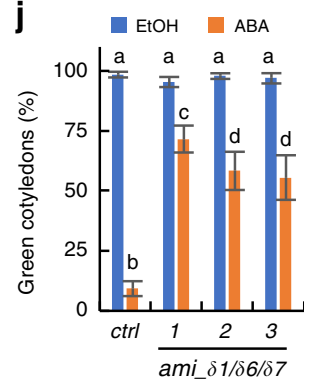

Fig. 5 MAPKK-kinases are required for plant ABA response. a Genome structures and T-DNA insertion sites of M3K genes are shown. $\mathbf{b}$ Genomic regions of CRISPR/Cas9-mediated M3K $\delta 1$ and $M 3 K \delta 7$ gene deletions are shown. These deletions were introduced in the $m 3 k \delta 6-2$ T-DNA knockout mutant as a background. $\mathbf{c}$ RT-PCR assays show transcripts of kinase domains of $M 3 K s$ in the $m 3 k \delta 1 c r i s p r ~ m 3 k \delta 6-2 m 3 k \delta 7 c r i s p r$ triple mutant. d $m 3 k \delta 1 c r i s p r$ $m 3 k \delta 6-2 m 3 k \delta 7$ crispr triple mutant seedlings were grown on $1 / 2$ MS plates supplemented with $2 \mu \mathrm{M}$ ABA or ethanol (control) for 16 days. e Seedlings showing green cotyledons as in $\mathbf{d}$ were counted. $n=3(\mathrm{EtOH})$ and $n=4$ $(A B A)$ experiments, means \pm s.d., 45 seeds per genotype were used in each experiment. f RT-PCR shows $M 3 K \delta 1, \delta 6$, and $\delta 7$ expression in the indicated $m 3 k$ T-DNA insertion mutants. $\delta 6(K D)$ refers to primers that amplify the

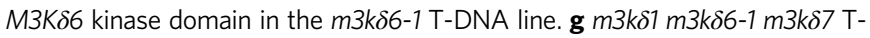
DNA triple mutant plants were grown on $1 / 2 \mathrm{MS}$ plates supplemented with $0.8 \mu \mathrm{M}$ ABA for 9 days. $\mathbf{h}$ Seedlings showing green cotyledons as in $\mathbf{g}$ were counted. $n=3$, means \pm s.d., $60-88$ seeds were used per genotype in each assay. $\mathbf{i}$ Three amiRNA lines targeting $M 3 \mathrm{~K} \delta 1, \delta 6$, and $\delta 7$ were grown on $1 /$ $2 \mathrm{MS}$ plates supplemented with $\mathrm{EtOH}$ (control) or $2 \mu \mathrm{M} \mathrm{ABA}$ for 9 days. As a control line, the amiRNA-HsMYO line ${ }^{21}$ was used. $\mathbf{j}$ Seedlings showing green cotyledons as in $\mathbf{i}$ were counted. $n=3$ (EtOH) and 4 (ABA) experiments, means \pm s.d., 81 seeds per genotype were analyzed in each experiment. (e, $\mathbf{h}$, and $\mathbf{i}$ ) Letters at the top of columns are grouped based on two-way ANOVA and Tukey's test, $P<0.05$.
Together these results support that these M3Ks have a function in ABA responses.

ABA- and osmotic stress- SnRK2 activations require M3Ks. Ingel kinase assays showed that ABA-induced activation of SnRK2 kinase in the $m 3 k \delta 1$ crispr $m 3 k \delta 6-2 m 3 k \delta 7$ crispr triple was slightly less strong than in wild-type plants (Fig. 6a, b; $n=4$ experiments). We further found a slightly reduced $\mathrm{ABA}$ activation of SnRK2 kinase activity in the T-DNA insertion $m 3 k \delta 1 m 3 k \delta 6-1$ $m 3 k \delta 7$ triple mutant compared to wild-type controls (Fig. $6 \mathrm{c}, \mathrm{d}$, Supplementary Fig. 12; $n=4$ experiments), similar to the $m 3 k \delta 1$ crispr $m 3 k \delta 6-2 m 3 k \delta 7 c r i s p r$ triple knockout mutant allele findings. Osmotic stress is known to rapidly activate OST1/ SnRK2.6 independent of ABA signalling ${ }^{20}$. Interestingly, we found that 15 min osmotic stress-induced SnRK2 activation was strongly impaired in these two independent $m 3 k$ triple mutant alleles, and this impairment was stronger than that in response to ABA application (Fig. 6a-d, Supplementary Fig. 12; $n=4$ experiments per allele).

In-gel kinase assays suggest that the M3Ks have a major role in osmotic stress signalling in Arabidopsis (Fig. 6a-d). We therefore investigated osmotic-stress responses of the $m 3 k$ double and triple mutants and the $m 3 k$ amiRNA lines, and found that they showed reduced sensitivity to osmotic-stress in seed germination assays (Supplementary Fig. 13). SnRK2 gene functions are highly redundant in mediating osmotic stress resistance ${ }^{34}$. At least nine members out of the ten Arabidopsis SnRK2 proteins are activated by osmotic stress through unknown mechanisms, while three members (SnRK2.2/2.3/2.6) are major ABA-activated SnRK2 $s^{19,23}$. In vitro in-gel kinase assays showed that $\mathrm{M} 3 \mathrm{~K} \delta 1$ strongly activated SnRK2.2 and 2.3 (Fig. 6e) as well as OST1/SnRK2.6 (Fig. 1g). SnRK2.3 was also activated by M3K $\delta 6$ and $\mathrm{M} 3 \mathrm{~K} \delta 7$ (Supplementary Fig. 14a). We also found that SnRK2.2 (S180A) and SnRK2.3 (S172A), which have a mutation corresponding to OST1/SnRK2.6 (S171A), are not activated by ABA in mesophyll cell protoplasts in contrast to WT SnRK2.2 and WT SnRK2.3 (Supplementary Fig. 14b). M3K $\delta 1$ also activated SnRK2.4 kinase in vitro that is known to be activated by osmotic stress ${ }^{19}$.

Co-immunoprecipitation of M3K $\delta 6$ - and OST1/SnRK2.6expressed in mesophyll cell protoplasts did not show a clear interaction (Supplementary Fig. 15a). Protein kinase interactions are often transient and do not show co-immunoprecipitation with their targets ${ }^{35}$. BiFC analyses can detect transient interactions in plant cells. Quantitative BiFC experiments provide evidence that

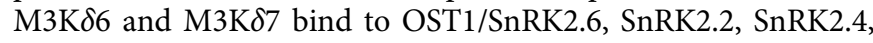
and SnRK2.10 in plant cells with different efficiencies (Supplementary Fig. 15b-e). We further observed that the M3K $\delta 6-F L A G$ protein band in SDS-PAGE gels was slightly shifted in response to 15 min osmotic stress treatment in mesophyll cell protoplasts, suggesting an osmotic stress-dependent post-translational modification of $\mathrm{M} 3 \mathrm{~K} \delta 6$ (Fig. 6f; $n=3$ ).

\section{Discussion}

In the present study, a combination of genetic screening for functional redundancy in abscisic acid responsiveness and multiple biochemical and signal transduction analyses in vitro and in planta have identified and characterized members of the Raf-like MAPKK-kinase $\delta$ B3 family that are required for full activation of SnRK2 protein kinases in abscisic acid signal transduction in vitro (Fig. $1 \mathrm{~g}$ and Fig. $4 \mathrm{a}-\mathrm{c}$ ), in a reconstitution system (Fig. $4 \mathrm{~d}-\mathrm{f}$ ) and in planta (Figs. $1 \mathrm{a}-\mathrm{e}, 5 \mathrm{~d}-\mathrm{j}$ and $6 \mathrm{a}-\mathrm{d}$ ). Triple mutants in the $M 3 \mathrm{Ks}$ At $M 3 K \delta 1, M 3 K \delta 6$, and $M 3 K \delta 7$ show impaired ABA- and osmotic stress-responses. As the Arabidopsis genome includes 80 MAPKK-kinases ${ }^{22}$, of which 22 MAPKK-kinases are in the B 
a

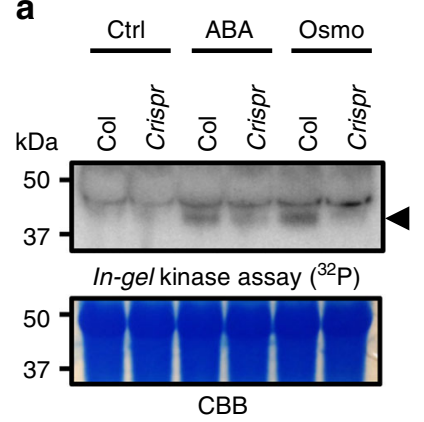

b

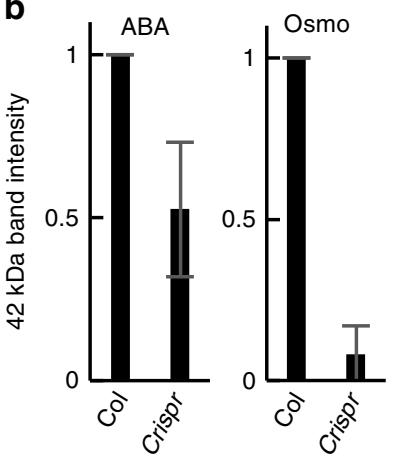

C

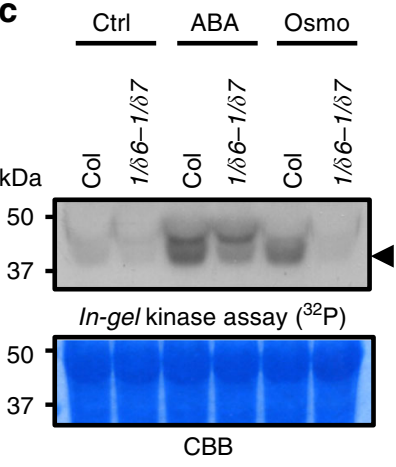

d
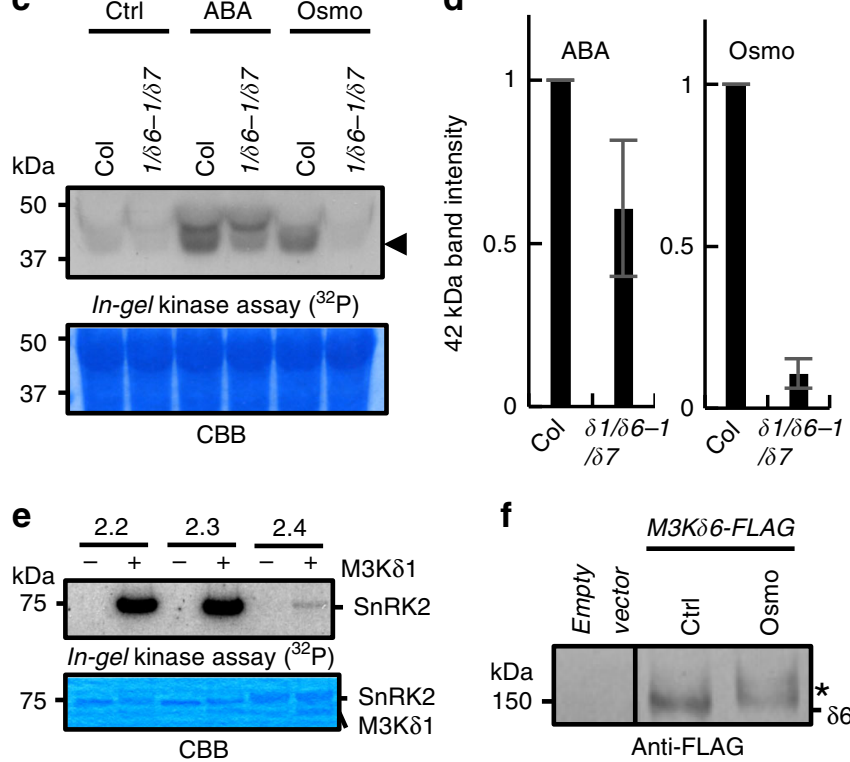

$\mathbf{f}$

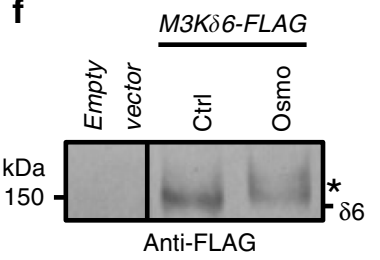

Fig. 6 MAPKK-kinases mediate ABA- and osmotic stress-induced SnRK2 activation in planta. a $m 3 k \delta 1 c r i s p r m 3 k \delta 6-2 m 3 k \delta 7 c r i s p r$ triple mutant seedlings were incubated in $10 \mu \mathrm{M} \mathrm{ABA}$ or $0.3 \mathrm{M}$ mannitol (Osmo) for 15 min. SnRK2 activities were tested by in-gel kinase assays. Arrowhead shows SnRK2 activity ${ }^{23}$. b Normalized band intensities as shown in a were measured by using ImageJ. $n=4$, means \pm s.e.m. c $m 3 k \delta 1 m 3 k \delta 6-1 m 3 k \delta 7$ TDNA triple mutant seedlings were incubated in $10 \mu \mathrm{M} \mathrm{ABA}$ or $0.3 \mathrm{M}$ mannitol (Osmo) for $15 \mathrm{~min}$. SnRK2 activities were analyzed by in-gel kinase assays. $\mathbf{d}$ Normalized band intensities as shown in $\mathbf{c}$ were measured by using ImageJ. $n=4$ experiments, means \pm s.e.m. e, Recombinant GSTtagged Arabidopsis SnRK2 protein kinases were incubated with $M 3 K \delta 1$ kinase domain. SnRK2 kinase activities were analyzed by in-gel kinase assays. $\mathbf{f} M 3 K \delta 6-F L A G$ was transiently expressed in Arabidopsis mesophyll cell protoplasts. Protoplasts were incubated in $0.8 \mathrm{M}$ mannitol (Osmo) for $15 \mathrm{~min} . \mathrm{M} 3 \mathrm{~K} \delta 6$ proteins were detected by immuno-blot using anti-FLAG antibody. In the Osmo lane, the M3K $\delta 6$ band showed a slight mobility shift as indicated by an asterisk.

subgroup, it is conceivable that additional members of this family contribute to ABA responses and that higher order mutants will cause enhanced ABA insensitivity. Previous studies suggest that other MAPKK-kinases, than those identified here, are involved in aspects of ABA signalling through a MAP3K-MAP2K-MAPK cascade $^{36-38}$ or through unknown pathways ${ }^{39,40}$.

Dephosphorylation of the OST1/SnRK2.6 kinase was unexpectedly found not to result in OST1/SnRK2.6 reactivation by

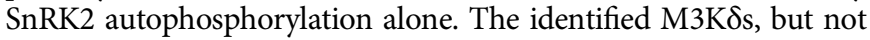
other analyzed CPK and MPK12 protein kinases that function in ABA signalling $24-27$, were found to be required for reactivation of OST1/SnRK2.6. Moreover, the M3K $\delta 1$ kinase greatly enhances the activities of other ABA signalling protein kinases SnRK2.2 and

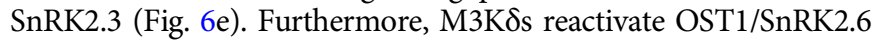
through phosphorylation of Ser171 in OST1/SnRK2.6. The Ser171 residue in OST1/SnRK2.6 is essential for ABA responses in planta (Fig. 3 and Supplementary Fig. 7), but OST1/SnRK2.6 cannot autophosphorylate this Ser-171 residue (Fig. 2) ${ }^{11,16}$. These data point to the model that the M3Kסs identified here are essential for SnRK2 kinase reactivation and thus robust $\mathrm{ABA}$ responses in plants. Higher order M3K mutants and further experiments will be needed to investigate M3K-dependent Ser171 phosphorylation of OST1/ SnRK2.6 in planta.

A previous proof-of-concept screen using artificial microRNAs that target multiple homologous genes isolated a plant predicted to target seven M3Ks of the B-family ${ }^{21}$. A Physcomitrella single gene encoding a $\mathrm{M} 3 \mathrm{~K}, \mathrm{ARK}$, was also identified which functions in SnRK2 activation ${ }^{28}$. Recent studies show that ARK kinase is required for Physcomitrella ABA and drought stress responses including phosphorylation of transcription factors through SnRK2 kinases ${ }^{41,42}$. Here, in forward genetic screening we have isolated amiRNA-expressing lines that target M3K members of the B family (Fig. 1a-c and Supplementary Fig. 1). In the present study, we show that for prior dephosphorylated SnRK2 kinases, we could robustly reconstitute ABAactivation of OST1/SnRK2.6 and the SLAC1 anion channel only in the presence of $\mathrm{M} 3 \mathrm{~K} \delta \mathrm{s}$ in vitro and in Xenopus oocytes (Fig. 4). The present experiments reveal that autophosphorylation cannot alone reactivate the SnRK2 kinases. These data suggest that these M3Ks are a missing component of the early ABA signalling module in plants.

Osmotic stress is known to rapidly activate SnRK2 protein kinases ${ }^{20,34,43}$. Rapid osmotic stress signalling includes a prominent ABA-independent pathway that leads to activation of transcription factors ${ }^{44,45}$. However, the upstream osmotic stress signalling mechanisms remain incompletely understood. Recent studies suggest that PP2Cs involved in ABA signalling dephosphorylate SnRK2.4 ${ }^{46-48}$. The M3K ARK is required for osmotic stress tolerance in Physcomitrella ${ }^{28,42}$. Interestingly, the identified $\mathrm{M} 3 \mathrm{~K} \delta$ s play a critical role in the rapid osmotic stress activation of SnRK2 protein kinases (Fig. 6a-d). In $m 3 k$ triple mutants, 15 min short term osmotic stress activation of SnRK2 is greatly impaired in planta. This impairment in rapid osmotic stress activation of SnRK2 protein kinases is prominent in the investigated $m 3 k \delta 1 /$ $\delta 6 / \delta 7$ triple mutant alleles, in contrast to that of $\mathrm{ABA}$ activation of SnRK2 kinases (Fig. 6a-d). Further research will be needed to determine whether higher order $m 3 k$ mutants further impair the ABA response. To start testing this hypothesis, we created $m 3 k \delta 1 /$ $\delta 5 / \delta 6-1 / \delta 7$ quadruple mutant plants and found that they show a stronger $\mathrm{ABA}$-insensitive phenotype in seed germination than the triple mutant (Supplementary Fig. 16a). Triple mutant plants, which include the weak allele $m 3 k \delta 6-1$ ( $m 3 k \delta 1 / \delta 6-1 / \delta 7)$, did not show a clear phenotype in ABA-induced stomatal closing using a robust method of gas exchange analyses. The public eFP Browser shows a prominent guard cell expression of $M 3 K \delta 5$ (Supplementary Fig. 16b). $M 3 K \delta 5$ is targeted by the $m 3 k$ amiRNA (Supplementary Fig. 1), which shows an ABA-insensitive stomatal closing (Supplementary Fig. 10a, b) and impairs ABA activation of S-type anion channels (Supplementary Fig. 10c-h). Higher order mutants will be required to further investigate $\mathrm{M} 3 \mathrm{~K}$ functions in ABA-induced stomatal closing. The requirement of $\mathrm{M} 3 \mathrm{~K} \delta$ s for the rapid osmotic stress response suggests that these $\mathrm{M} 3 \mathrm{~K} \delta$ s also mediate osmotic stress signal transduction before the slower onset of ABA concentration increase 4-6 h after exposure to osmotic stress ${ }^{49}$. These findings are consistent with previous observations of an ABA-independent osmotic stress-triggered SnRK2 signal transduction pathway $20,43,50$. The present study points to a model in which the identified $\mathrm{M} 3 \mathrm{~K} \delta$ protein kinases 
may act as a convergence point of rapid osmotic stress signalling and prolonged abscisic acid signal transduction.

Osmotic and salt stresses induce a rapid cytosolic $\mathrm{Ca}^{2+}$ increase ${ }^{51-54}$. An ABA-independent osmotic stress signalling pathway has been characterized that triggers rapid gene expression $^{44,55}$. Recent research shows that the Arabidopsis NGATHA1 transcription factor mediates the ensuing drought stress-induced $\mathrm{ABA}$ accumulation through enhanced expression of the ABA biosynthesis NINE-CIS-EPOXYCAROTENOID DIOXYGENASE, NCED $3^{56}$. Gel shift assays indicate that osmotic stress causes a rapid post-translational modification of M3K $\delta 6$ (Fig. 6f). Our results reveal a key component by which plants respond initially to osmotic stress before measurable stressinduced ABA concentration increases in roots. Furthermore, interestingly, $m 3 k$ amiRNA lines impair robust ABA activation of SnRK2 kinases in planta. Further research will be required to elucidate the presently unknown mechanisms between osmotic stress sensing and $\mathrm{M} 3 \mathrm{~K} \delta$-dependent activation of SnRK2 protein kinases.

\section{Methods}

Genetic screening for ABA response mutants. Using amiRNA libraries ${ }^{21}$, we screened amiRNA lines for ABA-insensitive seed germination phenotypes using 1/ 2 MS plate supplemented with $2 \mu \mathrm{M} \mathrm{ABA}{ }^{57}$. The underlying amiRNA sequences were identified from genomic DNA by PCR and sequencing ( $m 3 k$ amiRNA: $5^{\prime}$ TTGGAGCCATCCATTCAGCCG-3', amiR-ax1117: 5' - TCCAAAATCG-

CAAACCTTCAC- $\left.3^{\prime}\right)$. We used an amiRNA line targeting human myosin 2 gene (HsMYO2) as a control.

\section{In vitro dephosphorylation and phosphorylation assays. Ten microgram of} GST-OST1/SnRK2.6 proteins were bound to glutathione sepharose 4B beads and incubated with $30 \mathrm{U}$ CIAP for $2 \mathrm{~h}$ at room temperature. The beads were washed with T-TBS (50 mM Tris- $\mathrm{HCl} \mathrm{pH} 7.5,150 \mathrm{mM} \mathrm{NaCl}, 0.05 \%$ Tween-20) three times, and GST-OST1 protein was eluted with $30 \mu \mathrm{L}$ elution buffer $(50 \mathrm{mM}$ Tris$\mathrm{HCl} \mathrm{pH} \mathrm{8.0,10} \mathrm{mM} \mathrm{reduced} \mathrm{glutathione).} \mathrm{Five} \mathrm{microliter} \mathrm{of} \mathrm{GST-OST1} \mathrm{solution}$ was added in phosphorylation buffer $[50 \mathrm{mM}$ Tris- $\mathrm{HCl} \mathrm{pH} 7.5,10 \mathrm{mM} \mathrm{MgCl}$, $2 \mu \mathrm{M}$ free $\mathrm{Ca}^{2+}$ buffered by $1 \mathrm{mM}$ EGTA and $\mathrm{CaCl}_{2}$ (https://somapp.ucdmc ucdavis.edu/pharmacology/bers/maxchelator/CaMgATPEGTA-NIST.htm), 0.1\% Triton X-100, and $1 \mathrm{mM}$ DTT] with or without $1 \mu \mathrm{g}$ of the protein kinases CPK6, CPK23, MPK12 or $0.1 \mu \mathrm{g}$ of the indicated MAPKK kinases (M3Ks). The phosphorylation reactions were started by addition of $200 \mu \mathrm{M}$ ATP and $1 \mu \mathrm{Ci}\left[\gamma^{-}{ }^{32} \mathrm{P}\right]$ ATP. After $60 \mathrm{~min}$ incubation at room temperature, these reactions were stopped by addition of SDS-PAGE loading buffer. Note that the mobilities of recombinant and transgenic proteins in the present study depend on the linked tags. For example, the OST1/SnRK2.6 6xHis-tag also includes sequences including thrombin and enterokinase cleavage sites and restriction enzyme sites in the pET-30a(+) vector used for E.coli expression of OST1/SnRK2.6 in Figs. 1g, 4a and b. Primer sequences used for cloning in this study are provided in Supplementary Table 1

In-gel kinase assays. Fifteen to twenty Arabidopsis seedlings (7-9-day-old) grown on $1 / 2 \mathrm{MS}$ plates were treated with $10 \mu \mathrm{M}$ ABA or $0.3 \mathrm{M}$ mannitol for $15 \mathrm{~min}$ at room temperature and grinded with a pestle and mortar in $400 \mu \mathrm{L}$ extraction buffer $(50 \mathrm{mM}$ MOPS-KOH pH 7.5, $100 \mathrm{mM} \mathrm{NaCl}, 2.5 \mathrm{mM}$ EDTA, $10 \mathrm{mM} \mathrm{NaF}, 2 \mathrm{mM}$ dithiothreitol, $1 \mathrm{mM}$ phenylmethylsulfonyl fluoride, $10 \mu \mathrm{M}$ leupeptin) on ice. After $10 \mathrm{~min}$ centrifugation at $13,000 \times g$, the supernatants were transferred to new tubes, and proteins were precipitated by acetone precipitation. Proteins were dissolved in SDSPAGE loading buffer and separated in $9 \%$ acrylamide gels. In-gel kinase assays were performed as described previously ${ }^{58}$. In brief, gels were incubated in washing buffer (25 mM Tris- $\mathrm{HCl}$ pH 8.0, 0.5 mM DTT, $0.1 \mathrm{mM} \mathrm{Na}_{3} \mathrm{VO}_{4}, 5 \mathrm{mM} \mathrm{NaF}, 0.5 \mathrm{mg} \mathrm{ml}^{-1}$ BSA, and $0.1 \%$ Triton $\mathrm{X}-100$ ) for $30 \mathrm{~min}$ three times and in renaturation buffer (25 mM Tris- $\mathrm{HCl} \mathrm{pH} \mathrm{8.0,1} \mathrm{mM} \mathrm{DTT,} 0.1 \mathrm{mM} \mathrm{Na}_{3} \mathrm{VO}_{4}$, and $5 \mathrm{mM} \mathrm{NaF}$ ) for $30 \mathrm{~min}$ once. Gels were further incubated in renaturation buffer at $4{ }^{\circ} \mathrm{C}$ overnight followed by further incubation in reaction buffer $(50 \mathrm{mM}$ Tris- $\mathrm{HCl} \mathrm{pH} 7.5,10 \mathrm{mM} \mathrm{MgCl}, 2 \mathrm{mM}$ DTT, and $1 \mathrm{mM}$ EGTA) for $30 \mathrm{~min}$. Phosphorylation reactions were carried out in reaction buffer with $50 \mu \mathrm{Ci}\left[\gamma^{-32} \mathrm{P}\right]$-ATP for $60 \mathrm{~min}$ at room temperature. Gels were washed in $5 \%$ trichloroacetic acid and $1 \%$ phosphoric acid four times for $30 \mathrm{~min}$ each. Storage phosphor screens or X-ray films were used for detection.

In vitro reconstitution of ABA signalling. $0.43 \mu \mathrm{mol}$ His-OST1/SnRK2.6, 0.17 $\mu \mathrm{mol}$ His-PYR1/RCAR1 1 and $0.06 \mu \mathrm{mol}$ GST-M3K $\delta 6$ kinase domain were incubated in $200 \mu \mathrm{L}$ phosphorylation buffer $\left(50 \mathrm{mM}\right.$ Tris- $\mathrm{HCl} \mathrm{pH} 7.5,10 \mathrm{mM} \mathrm{MgCl}_{2}$, $0.1 \%$ Triton X-100, and $1 \mathrm{mM}$ DTT) with $200 \mu \mathrm{M}$ ATP for $10 \mathrm{~min}$, and $20 \mu \mathrm{L}$ solution was transferred to a new tube and $10 \mu \mathrm{L} 3 \mathrm{xSDS}$-PAGE loading buffer was added to stop the reaction. Then, $0.01 \mu$ mol His-HAB1 was added to the reaction solution, and $20 \mu \mathrm{L}$ solution were transferred to a new tube to stop the reaction by addition of $10 \mu \mathrm{L} 3 \mathrm{xSDS}$-PAGE loading buffer after 10 min incubation. $50 \mu \mathrm{M}$ ABA was added to the reaction and $20 \mu \mathrm{L}$ reactions were transferred to new tubes to stop the reaction after 5,10 , or $30 \mathrm{~min}$ incubation. Proteins were separated by SDSPAGE, and OST1/SnRK2.6 activity was detected by in-gel kinase assays.

Identification of OST1/SnRK2.6 phosphorylation sites. Thirty microgram GSTOST1/SnRK2.6(D140A) and $2.5 \mu \mathrm{g}$ GST-M3K $\delta 1$ kinase domain were incubated in phosphorylation buffer $\left(50 \mathrm{mM}\right.$ Tris- $\mathrm{HCl}$ pH 7.5, $10 \mathrm{mM} \mathrm{MgCl}_{2}, 0.1 \%$ Triton X100 , and $1 \mathrm{mM}$ DTT) with $1 \mathrm{mM} \mathrm{ATP}$ for $2 \mathrm{~h}$ at room temperature. Proteins were precipitated by acetone precipitation and dissolved in SDS-PAGE loading buffer. After SDS-PAGE and CBB staining, protein bands of GST-OST1/SnRK2.6(D140A) were excised and analyzed by LC-MS/MS ${ }^{17}$. For in vivo Ser-171 phosphorylation OST1/SnRK2.6-GFP was transiently expressed in Arabidopsis mesophyll cell protoplasts. The protoplasts were incubated with or without $20 \mu \mathrm{M}$ ABA for $15 \mathrm{~min}$ and OST1/SnRK2.6 proteins were purified by immunoprecipitation using anti-GFP antibodies. After SDS-PAGE and CBB staining, OST1/SnRK2.6-GFP bands were excised and analyzed by LC-MS/MS ${ }^{17}$

Analysis of stomatal ABA response. Infrared-based gas exchange analyzer systems were used including an integrated Multiphase Flash Fluorometer (Li-680001A or Li-6400; Li-Cor Inc.) for gas exchange analyses. Plants were grown on soil in Percival growth cabinets at a $12 / 12 \mathrm{~h}, 21^{\circ} \mathrm{C} / 21^{\circ} \mathrm{C}$ day/night cycle, a photosynthetic photon flux density of $\sim 90 \mathrm{mmol} \mathrm{m}^{-2} \mathrm{~s}^{-1}$, and $70-80 \%$ relative humidity for 6-7 weeks. Mature rosette leaves were detached at the basal part of petiole by a razor blade, and re-cut twice under distilled and deionized water. The petioles of the leaves were then immersed in $\mathrm{ddH} 2 \mathrm{O}$ for gas exchange analysis. The detached leaves were clamped and the environment of the leaf chamber was controlled at 400 ppm ambient $\mathrm{CO}_{2}, 23 \sim 24^{\circ} \mathrm{C}, \sim 65 \%$ relative air humidity, $150 \mu \mathrm{mol} \mathrm{m}{ }^{-2} \mathrm{~s}^{-1}$ photon flux density, and $500 \mu \mathrm{mol} \mathrm{s} \mathrm{s}^{-1}$ flow rate until stomatal conductance stabilized. One or $2 \mu \mathrm{M} \pm-\mathrm{ABA}$ was applied to the petiole for kinetic stomatal conductance response analyses as described ${ }^{59}$.

Patch-clamp analyses. Guard cell protoplasts from 4 to 6-week-old Arabidopsis plants were prepared ${ }^{24,33}$. ABA-activated S-type anion channel current recordings were carried out by using an Axon $200 \mathrm{~A}$ amplifier (Axon instruments) and a Digidata 1440 A low-noise data acquisition system. Epidermal tissues were isolated from one or two rosette leaves and collected using a nylon mesh $(100-\mu \mathrm{m}$ pore size). Subsequently the epidermal tissues were incubated in $10-\mathrm{ml}$ protoplast isolation solution containing $500 \mathrm{mM}$ D-mannitol, 1\% cellulase R-10 (Yakult Pharmaceutical Industry), 0.5\% macerozyme R-10 (Yakult Pharmaceutical Industry), $0.5 \%$ bovine serum albumin, $0.1 \%$ kanamycin sulfate, $0.1 \mathrm{mM} \mathrm{CaCl}_{2}, 0.1 \mathrm{mM} \mathrm{KCl}$ and $10 \mathrm{mM}$ ascorbic acid for $16 \mathrm{~h}$ at $25^{\circ} \mathrm{C}$ on a circular shaker at $50 \mathrm{rpm}$. Guard cell protoplasts were collected through a nylon mesh $(10-\mu \mathrm{m}$ pore size) and then washed two times with protoplast suspension solution containing $500 \mathrm{mM} \mathrm{D}$ sorbitol, $0.1 \mathrm{mM} \mathrm{CaCl}_{2}$, and $0.1 \mathrm{mM} \mathrm{KCl} \mathrm{(pH} 5.6$ with $\left.\mathrm{KOH}\right)$ by centrifugation $(200 \times g$ for $5 \mathrm{~min}$ at room temperature). Isolated guard cell protoplasts were stored on ice before use.

S-type anion currents in guard cell protoplasts were recorded using the wholecell patch-clamp technique $\mathrm{e}^{24,33}$. The pipette solution was composed of $150 \mathrm{mM}$ $\mathrm{CsCl}, 2 \mathrm{mM} \mathrm{MgCl}_{2}, 5.86 \mathrm{mM} \mathrm{CaCl}_{2}, 6.7 \mathrm{mM}$ EGTA, and $10 \mathrm{mM}$ Hepes-Tris (pH 7.1). $5 \mathrm{mM} \mathrm{Mg-ATP}$ was added to the pipette solution freshly before use. The bath solution was composed of $30 \mathrm{mM} \mathrm{CsCl}, 2 \mathrm{mM} \mathrm{MgCl}_{2}, 1 \mathrm{mM} \mathrm{CaCl}_{2}$, and $10 \mathrm{mM}$ MES-Tris ( $\mathrm{pH}$ 5.6). Osmolalities of the pipette solution and the bath solution were adjusted to 500 mosmol kg-1 and 485 mosmol kg-1 using D-sorbitol, respectively. In Fig. 3, guard cell protoplasts were pre-incubated for $20 \mathrm{~min}$ in the bath solution containing $50 \mu \mathrm{M}$ ABA prior to recordings, and $\mathrm{ABA}$ was added to the pipette solution. In Supplementary Fig. 10, guard cell protoplasts were pre-incubated for $30 \mathrm{~min}$ in the bath solution containing $10 \mu \mathrm{M}$ ABA prior to recordings.

Two-electrode voltage clamp recordings. The PCR amplified cDNA fragments of OST1, SLAC1, PYL9/RCAR1, ABI1, M3K $\delta 1, \mathrm{M} 3 \mathrm{~K} \delta 6$, and M3K $\delta 7$ were cloned into the oocyte expression vector pNB1 by using an advanced uracil-excision based cloning strategy as previously described ${ }^{60}$. The mutant isoforms OST1-S171A, M3K $86-\mathrm{K} 775 \mathrm{~W}$, and M3K $87-\mathrm{K} 740 \mathrm{~W}$ were generated using the Quikchange SiteDirected Mutagenesis kit (Agilent Technologies). Linearized plasmids were used to generate cRNAs via the mMESSAGE mMACHINE ${ }^{\oplus}$ T7 kit (Thermo Fisher Scientific, Catalog number: AM1344). Surgically extracted ovaries of Xenopus laevis were ordered from Nasco (Fort Atkinson, Wisconsin, product number: LM00935) and Ecocyte Bio Science US (Austin, Texas) and oocytes were isolated as previously described $^{61}$. Five nanogram of cRNA of each construct OST1, OST1-S171A, SLAC1, PYL9/RCAR1, ABI1 and $0.5 \mathrm{ng}$ cRNAs of each construct $M 3 K \delta 1, M 3 K \delta 6, M 3 K \delta 7$ $M 3 K \delta 6-K 775 W, M 3 K \delta 7-K 740 W$ were co-injected into isolated oocytes in the indicated combinations. Oocytes were then incubated at $16^{\circ} \mathrm{C}$ for 2 days in ND96 buffer ( $1 \mathrm{mM} \mathrm{CaCl}_{2}, 1 \mathrm{mM} \mathrm{MgCl}$, $96 \mathrm{mM} \mathrm{NaCl}, 10 \mathrm{mM} \mathrm{MES/Tris,} \mathrm{pH}=7.5$ ). Osmolarity was adjusted to $220 \mathrm{mosmol} \mathrm{kg} \mathrm{k}^{-1}$ by D-sorbitol. Using a Cornerstone (Dagan) TEV-200 amplifier and a Digidata 1440 A low-noise data acquisition system with pClamp software (Molecular Devices), two-electrode voltage clamp recordings were performed in a bath solution containing $1 \mathrm{mM} \mathrm{CaCl}_{2}, 2 \mathrm{mM} \mathrm{KCl}$, $24 \mathrm{mM} \mathrm{NaCl}, 70 \mathrm{mM}$ Na-gluconate, $10 \mathrm{mM}$ MES/Tris, $\mathrm{pH}$ 7.4, Osmolarity was 
adjusted to 220 mosmol $\mathrm{kg}^{-1}$ by D-sorbitol. ABA was injected into oocytes to achieve a final concentration of $50 \mu \mathrm{M}$ for analyses of ABA activation of SLAC1 currents. Steady-state currents were recorded with $3 \mathrm{~s}$ voltage pulses ranging from $+40 \mathrm{mV}$ to $-120 \mathrm{mV}$ in $-20 \mathrm{mV}$ decrements, followed by a "tail" voltage of -120 $\mathrm{mV}$ and the holding potential was kept at $0 \mathrm{mV}$.

SLAC1-mediated currents in oocytes vary showing either time-dependent relaxation or more instantaneous currents when using a chloride bath solution ${ }^{61,62}$. Furthermore, ion channel activities display different magnitudes from one oocyte batch to another due to protein expression level variation among batches of oocytes. To avoid time-of-measurement and inter-batch dependence in the data, $\mathrm{H}_{2} \mathrm{O}$-injected control and other indicated controls were included in each batch of oocytes and control experiments were recorded intermittently with the investigated conditions. Data from one representative oocyte batch are shown from the same batch in each figure panel and at least three independent batches of oocytes were investigated and showed consistent findings.

Mesophyll cell protoplast assays. Mesophyll cell protoplasts were isolated as described previously ${ }^{63}$ from 3-4-week-old Arabidopsis leaves. 10-20 $\mu \mathrm{g}$ of pUC18 plasmids carrying 35 S:OST1/SnRK2.6-GFP:nosT or 35 S:M3K86-FLAG:nosT and $30 \mu \mathrm{g}$ protoplasts were used for $20 \%$ PEG-mediated transient expression. After overnight incubation in incubation buffer $(10 \mathrm{mM}$ MES-KOH $\mathrm{pH} 6.0,0.4 \mathrm{M}$ mannitol, $20 \mathrm{mM} \mathrm{KCl}, 1 \mathrm{mM} \mathrm{CaCl} 2$ ), protoplasts were incubated in $10 \mu \mathrm{M} \mathrm{ABA}$ or $0.8 \mathrm{M}$ mannitol or in control buffer for $15 \mathrm{~min}$ and harvested by centrifugation at $13,000 \times g$ for $1 \mathrm{~min}$. After the supernatants were removed, $20 \mu \mathrm{L}$ SDS-PAGE loading buffer was added and incubated at $95^{\circ} \mathrm{C}$ for $3 \mathrm{~min}$.

\section{Measurements of leaf temperatures by thermal imaging. Plants grown} 4-5 weeks on soil were sprayed with $20 \mu \mathrm{M}$ ABA dissolved in water. After $3 \mathrm{~h}$ under white light in the growth room, images were captured using an infrared thermal imaging camera (T650sc; FLIR, Wilsonville, Oregon). Leaf temperatures were determined as average temperatures of each whole leaf area by using Fiji software (ImageJ version: $2.0 .0-\mathrm{rc}-59 / 1.51 \mathrm{n}$ ).

Creating CRISPR/Cas9-based knockout Arabidopsis. The $m 3 k \delta 1$ and $m 3 k \delta 7$ CRISPR/Cas9 deletion knockout mutants were generated using CRISPR/Cas9 gene editing technology ${ }^{64-66}$ in the $m 3 k \delta 6-2$ mutant background. We used two guide RNAs to generate a large deletion in each target gene. The target sequences in $M 3 K \delta 1$ were TACGGAAGCTCCACATCGGCGG and GATGCAAGTCGTTGG AGCTGTGG (PAM sites are underlined). Targets for $M 3 K \delta 7$ were GACGGAG TTCCA $\overline{G A T C T C C G G G}$ and CCAGAGAGCAGCAGTTCCCAGT.

The designed $m \overline{3 k \delta 1 c r i s p r}$ mutants were genotyped with the primer pair Delta1GT1 and Delta1-GT2, which would generate a fragment of about $750 \mathrm{bp}$ when the designed deletion took place. The primer pair could not amplify WT genomic DNA due to the large size of the fragment. To determine zygosity of $m 3 k \delta 1 \mathrm{crispr}$ mutants, we used the primer set Delta1-GT1 + Delta1-GT3, which amplifies a 777 bp fragment from WT DNA, but could not amplify a band in a homozygous mutant.

For $m 3 k \delta 7$ crispr mutants, we used Delta7-GT1 and Delta7-GT4, which would generate a fragment of about $1390 \mathrm{bp}$ if mutant DNA is used as PCR template. The primer pair could not amplify WT DNA because of the large fragment size. The Delta7-GT1 and Delta7-GT3 primer pair was able to generate a fragment of 1125 bp when WT DNA was used as PCR template. The Delta7-GT1/GT3 was used to differentiate homozygous $m 3 k \delta 7$ crispr mutants from heterozygous $m 3 k \delta 7$ crispr mutants. After isolating homozygous $m 3 k \delta 1$ crispr $m 3 k \delta 6-2$ and $m 3 k \delta 7 c r i s p r$ $m 3 k \delta 6-2$ mutants, these lines were crossed and homozygous triple mutants were recovered in the T2 generation. Primers for genotyping: Deltal-GT1: $5^{\prime}-$ TTGTTGGTTCCACGAACGGA-3', Delta1-GT2: 5'-GATGGCCGTAAAT GCGGTTC-3', Delta1-GT3: 5'-CGGATCAGGATCAGAGACGC-3', Delta7-GT1: 5'-TGCATAAGGTGGTGAGCGAA-3', Delta7-GT3: 5'-CCAAACCCTGCA TCCCAGAT-3', Delta7-GT4: 5'-GTCAAGGAAGAAGCGACCCA-3'

Creating amiRNA knock-downs targeting $\boldsymbol{M} 3 \boldsymbol{K} \boldsymbol{\delta} \mathbf{1}, \boldsymbol{\delta} \boldsymbol{6}$ and $\boldsymbol{\delta} \mathbf{7}$. The amiRNA sequence was designed using the WMD3 (http://wmd3.weigelworld.org/cgi-bin/ webapp.cgi) and PHANTOM database (http://phantomdb.ucsd.edu). The amiRNA containing the target sequence (5'-TACGACTTGCATCGGGTTCAA-3') for $M 3 K \delta 1, M 3 K \delta 6$, and $M 3 K \delta 7$ was amplified by PCR using primers (I: $5^{\prime}$-gaTACG ACTTGCATCGGGTTCAAtctctcttttgtattcc-3', II: 5' -gaTTGAACCCGATGCAAG TCGTAtcaaagagaatcaatga-3',

III: 5'-gaTTAAACCCGATGCTAGTCGTTtcacaggtcgtgatatg-3',

IV: 5'-gaAACGACTAGCATCGGGTTTAAtctacatatatattcct-3'), and inserted into the vector $p F H 0032^{21}$. Arabidopsis (Col-0) plants were used for floral-dip transformation. Three independent homozygous T3 seeds were used in the seed germination assays.

BiFC analyses. Constructs for $\mathrm{BiFC}$ analyses were generated by ligation of coding sequences of ABI1, RopGEF1, OST1/SnRK2.6, SnRK2.2, SnRK2.4, SnRK2.10, $\mathrm{M} 3 \mathrm{~K} \delta 6$, and $\mathrm{M} 3 \mathrm{~K} \delta 7$ into $\mathrm{pSPYCE}(\mathrm{M})$ or pSPYNE173 using the USER Cloning technology (see Supplementary Table 1 for primer sequences). Plasmids were transformed into Agrobacterium tumefasciens (GV3101) and co-infiltrated with a plasmid expressing the silencing suppressor p19 in leaves of 6-week-old Nicotiana benthamiana plants. Subcellular localization analyses were performed using a Nikon Eclipse TE2000-U confocal microscope. Images were acquired using a Plan Apo VC 60XA/1.20 WI objective using identical settings (exposure time and gain). Three independent experiments were conducted where three leaves were analyzed for each combination. $5 \mathrm{z}$-stacks were acquired for each leaf. Maximum projections of $\mathrm{z}$-stacks for each BiFC combination were quantified using Fiji and normalized over an infiltration control expressing p19 only.

Statistics. Cotyledon greening assays were analyzed by two-way ANOVA followed by Tukey's tests. Leaf temperatures were analyzed by one-way ANOVA followed by Tukey's tests.

Reporting summary. Further information on research design is available in the Nature Research Reporting Summary linked to this article.

\section{Data availability}

Arabidopsis mutants and transgenic lines used in this study are available upon request from the corresponding author. The source data for Figs. 1, 3-6, Supplementary Figs. $7-$ $11,13,15$ and 16 are provided as a Source Data file.

Received: 29 May 2019; Accepted: 4 December 2019; Published online: 02 January 2020

\section{References}

1. Finkelstein, R. Abscisic Acid synthesis and response. Arabidopsis Book 11, e0166 (2013).

2. Cutler, S. R., Rodriguez, P. L., Finkelstein, R. R. \& Abrams, S. R. Abscisic acid: emergence of a core signaling network. Annu Rev. Plant Biol. 61, 651-679 (2010).

3. Raghavendra, A. S., Gonugunta, V. K., Christmann, A. \& Grill, E. ABA perception and signalling. Trends Plant Sci. 15, 395-401 (2010).

4. Ma, Y. et al. Regulators of PP2C phosphatase activity function as abscisic acid sensors. Science 324, 1064-1068 (2009).

5. Park, S. Y. et al. Abscisic acid inhibits type $2 \mathrm{C}$ protein phosphatases via the PYR/PYL family of START proteins. Science 324, 1068-1071 (2009).

6. Weiner, J. J., Peterson, F. C., Volkman, B. F. \& Cutler, S. R. Structural and functional insights into core ABA signaling. Curr. Opin. Plant Biol. 13, 495-502 (2010).

7. Joshi-Saha, A., Valon, C. \& Leung, J. Abscisic acid signal off the STARting block. Mol. Plant 4, 562-580 (2011).

8. Tischer, S. V. et al. Combinatorial interaction network of abscisic acid receptors and coreceptors from Arabidopsis thaliana. Proc. Natl Acad. Sci. USA 114, 10280-10285 (2017).

9. Vlad, F. et al. Protein phosphatases $2 \mathrm{C}$ regulate the activation of the Snf1related kinase OST1 by abscisic acid in Arabidopsis. Plant Cell 21, 3170-3184 (2009).

10. Umezawa, $\mathrm{T}$. et al. Type $2 \mathrm{C}$ protein phosphatases directly regulate abscisic acid-activated protein kinases in Arabidopsis. Proc. Natl Acad. Sci. USA 106, 17588-17593 (2009).

11. Vlad, F. et al. Phospho-site mapping, genetic and in planta activation studies reveal key aspects of the different phosphorylation mechanisms involved in activation of SnRK2s. Plant J. 63, 778-790 (2010).

12. Geiger, D. et al. Activity of guard cell anion channel SLAC1 is controlled by drought-stress signaling kinase-phosphatase pair. Proc. Natl Acad. Sci. USA 106, 21425-21430 (2009).

13. Lee, S. C., Lan, W., Buchanan, B. B. \& Luan, S. A protein kinase-phosphatase pair interacts with an ion channel to regulate ABA signaling in plant guard cells. Proc. Natl Acad. Sci. USA 106, 21419-21424 (2009).

14. Fujii, H. et al. In vitro reconstitution of an abscisic acid signalling pathway. Nature 462, 660-664 (2009)

15. Takahashi, Y. et al. bHLH transcription factors that facilitate $\mathrm{K}^{+}$uptake during stomatal opening are repressed by abscisic acid through phosphorylation. Sci. Signal 6, ra48 (2013).

16. Belin, C. et al. Identification of features regulating OST1 kinase activity and OST1 function in guard cells. Plant Physiol. 141, 1316-1327 (2006).

17. Brandt, B. et al. Reconstitution of abscisic acid activation of SLAC1 anion channel by CPK6 and OST1 kinases and branched ABI1 PP2C phosphatase action. Proc. Natl Acad. Sci. USA 109, 10593-10598 (2012).

18. Takahashi, Y., Ebisu, Y. \& Shimazaki, K. I. Reconstitution of Abscisic Acid Signaling from the Receptor to DNA via bHLH Transcription Factors. Plant Physiol. 174, 815-822 (2017).

19. Boudsocq, M., Barbier-Brygoo, H. \& Laurière, C. Identification of nine sucrose nonfermenting 1-related protein kinases 2 activated by hyperosmotic and 
saline stresses in Arabidopsis thaliana. J. Biol. Chem. 279, 41758-41766 (2004).

20. Yoshida, R. et al. The regulatory domain of SRK2E/OST1/SnRK2.6 interacts with ABI1 and integrates abscisic acid (ABA) and osmotic stress signals controlling stomatal closure in Arabidopsis. J. Biol. Chem. 281, 5310-5318 (2006).

21. Hauser, F. et al. A Genomic-Scale Artificial MicroRNA Library as a Tool to Investigate the Functionally Redundant Gene Space in Arabidopsis. Plant Cell 25, 2848-2863 (2013).

22. MAPK, Group Mitogen-activated protein kinase cascades in plants: a new nomenclature. Trends Plant Sci. 7, 301-308 (2002).

23. Fujii, H. \& Zhu, J. K. Arabidopsis mutant deficient in 3 abscisic acid-activated protein kinases reveals critical roles in growth, reproduction, and stress. Proc. Natl Acad. Sci. USA 106, 8380-8385 (2009).

24. Brandt, B. et al. Calcium specificity signaling mechanisms in abscisic acid signal transduction in Arabidopsis guard cells. elife https://doi.org/10.7554/ eLife.03599 (2015).

25. Geiger, D. et al. Guard cell anion channel SLAC1 is regulated by CDPK protein kinases with distinct Ca2+ affinities. Proc. Natl Acad. Sci. USA 107, 8023-8028 (2010).

26. Jammes, F. et al. MAP kinases MPK9 and MPK12 are preferentially expressed in guard cells and positively regulate ROS-mediated ABA signaling. Proc. Natl Acad. Sci. USA 106, 20520-20525 (2009).

27. Des Marais, D. L. et al. Variation in MPK12 affects water use efficiency in Arabidopsis and reveals a pleiotropic link between guard cell size and ABA response. Proc. Natl Acad. Sci. USA 111, 2836-2841 (2014).

28. Saruhashi, M. et al. Plant Raf-like kinase integrates abscisic acid and hyperosmotic stress signaling upstream of SNF1-related protein kinase2. Proc. Natl Acad. Sci. USA 112, E6388-E6396 (2015).

29. Yoshida, R. et al. ABA-activated SnRK2 protein kinase is required for dehydration stress signaling in Arabidopsis. Plant Cell Physiol. 43, 1473-1483 (2002).

30. Mustilli, A. C., Merlot, S., Vavasseur, A., Fenzi, F. \& Giraudat, J. Arabidopsis OST1 protein kinase mediates the regulation of stomatal aperture by abscisic acid and acts upstream of reactive oxygen species production. Plant Cell 14, 3089-3099 (2002).

31. Laanemets, K. et al. Calcium-dependent and -independent stomatal signaling network and compensatory feedback control of stomatal opening via $\mathrm{Ca} 2+$ sensitivity priming. Plant Physiol. 163, 504-513 (2013).

32. Laanemets, $\mathrm{K}$. et al. Mutations in the SLAC1 anion channel slow stomatal opening and severely reduce $\mathrm{K}+$ uptake channel activity via enhanced cytosolic $[\mathrm{Ca} 2+]$ and increased $\mathrm{Ca} 2+$ sensitivity of $\mathrm{K}+$ uptake channels. $N$. Phytol. 197, 88-98 (2013).

33. Pei, Z. M., Kuchitsu, K., Ward, J. M., Schwarz, M. \& Schroeder, J. I. Differential abscisic acid regulation of guard cell slow anion channels in Arabidopsis wild-type and abi1 and abi2 mutants. Plant Cell 9, 409-423 (1997).

34. Fujii, H., Verslues, P. E. \& Zhu, J. K. Arabidopsis decuple mutant reveals the importance of SnRK2 kinases in osmotic stress responses in vivo. Proc. Natl Acad. Sci. USA 108, 1717-1722 (2011).

35. de Oliveira, P. S. et al. Revisiting protein kinase-substrate interactions: Toward therapeutic development. Sci. Signal 9, re3 (2016).

36. Danquah, A. et al. Identification and characterization of an ABA-activated MAP kinase cascade in Arabidopsis thaliana. Plant J. 82, 232-244 (2015).

37. Matsuoka, D., Yasufuku, T., Furuya, T. \& Nanmori, T. An abscisic acid inducible Arabidopsis MAPKKK, MAPKKK18 regulates leaf senescence via its kinase activity. Plant Mol. Biol. 87, 565-575 (2015).

38. Li, K. et al. AIK1, a mitogen-activated protein kinase, modulates abscisic acid responses through the MKK5-MPK6 kinase cascade. Plant Physiol. 173, 1391-1408 (2017).

39. Shitamichi, N., Matsuoka, D., Sasayama, D., Furuya, T. \& Nanmori, T. Overexpression of MAP3K delta 4, an ABA-inducible Raf-like MAP3K that confers salt tolerance in Arabidopsis. Plant. Plant Biotechnol. 30, 111-118 (2013).

40. Lee, S., Lee, M., Kim, J. \& Kim, S. Arabidopsis putative MAP kinase kinase kinases Raf10 and Raf11 are positive regulators of seed dormancy and ABA response. Plant Cell Physiol. 56, 84-97 (2015).

41. Amagai, A. et al. Phosphoproteomic profiling reveals ABA-responsive phosphosignaling pathways in Physcomitrella patens. Plant J. 94, 699-708 (2018).

42. Shinozawa, A. et al. SnRK2 protein kinases represent an ancient system in plants for adaptation to a terrestrial environment. Commun. Biol. 2, 30 (2019).

43. Boudsocq, M., Droillard, M. J., Barbier-Brygoo, H. \& Laurière, C. Different phosphorylation mechanisms are involved in the activation of sucrose nonfermenting 1 related protein kinases 2 by osmotic stresses and abscisic acid. Plant Mol. Biol. 63, 491-503 (2007).

44. Yamaguchi-Shinozaki, K. \& Shinozaki, K. Transcriptional regulatory networks in cellular responses and tolerance to dehydration and cold stresses. Annu Rev. Plant Biol. 57, 781-803 (2006).
45. Liu, Q. et al. Two transcription factors, DREB1 and DREB2, with an EREBP/ AP2 DNA binding domain separate two cellular signal transduction pathways in drought- and low-temperature-responsive gene expression, respectively, in Arabidopsis. Plant Cell 10, 1391-1406 (1998).

46. Krzywińska, E. et al. Phosphatase ABI1 and okadaic acid-sensitive phosphoprotein phosphatases inhibit salt stress-activated SnRK2.4 kinase. BMC Plant Biol. 16, 136 (2016).

47. Krzywińska, E. et al. Protein phosphatase type 2C PP2CA together with ABI1 inhibits SnRK2.4 activity and regulates plant responses to salinity. Plant Signal Behav. 11, e1253647 (2016).

48. Ruschhaupt, M. et al. Rebuilding core abscisic acid signaling pathways of Arabidopsis in yeast. EMBO J. 38, e101859 (2019).

49. Waadt, R. et al. FRET-based reporters for the direct visualization of abscisic acid concentration changes and distribution in Arabidopsis. eLife 3, e01739 (2014).

50. Zhao, Y. et al. Arabidopsis duodecuple mutant of PYL ABA receptors reveals PYL repression of ABA-independent SnRK2 activity. Cell Rep. 23, 3340-3351 (2018). e3345.

51. Yuan, F. et al. OSCA1 mediates osmotic-stress-evoked $\mathrm{Ca} 2+$ increases vital for osmosensing in Arabidopsis. Nature 514, 367-371 (2014).

52. Choi, W. G., Toyota, M., Kim, S. H., Hilleary, R. \& Gilroy, S. Salt stressinduced $\mathrm{Ca} 2+$ waves are associated with rapid, long-distance root-to-shoot signaling in plants. Proc. Natl Acad. Sci. USA 111, 6497-6502 (2014).

53. Stephan, A. B., Kunz, H. H., Yang, E. \& Schroeder, J. I. Rapid hyperosmoticinduced $\mathrm{Ca} 2+$ responses in Arabidopsis thaliana exhibit sensory potentiation and involvement of plastidial KEA transporters. Proc. Natl Acad. Sci. USA 113, E5242-E5249 (2016).

54. Kudla, J. et al. Advances and current challenges in calcium signaling. $N$. Phytol. 218, 414-431 (2018).

55. Yoshida, T., Mogami, J. \& Yamaguchi-Shinozaki, K. ABA-dependent and ABA-independent signaling in response to osmotic stress in plants. Curr. Opin. Plant Biol. 21, 133-139 (2014).

56. Sato, $\mathrm{H}$. et al. Arabidopsis thaliana NGATHA1 transcription factor induces ABA biosynthesis by activating NCED3 gene during dehydration stress. Proc. Natl Acad. Sci. USA 115, E11178-E11187 (2018).

57. Hauser, F. et al. A seed resource for screening functionally redundant genes and isolation of new mutants impaired in $\mathrm{CO} 2$ and $\mathrm{ABA}$ responses. J. Exp. Bot. 70, 641-651 (2019).

58. Hsu, P. K. et al. Abscisic acid-independent stomatal $\mathrm{CO}_{2}$ signal transduction pathway and convergence of $\mathrm{CO}_{2}$ and ABA signaling downstream of OST1 kinase. Proc. Natl Acad. Sci. USA 115, E9971-E9980 (2018).

59. Ceciliato, P. et al. Intact leaf gas exchange provides a robust method for measuring the kinetics of stomatal conductance responses to abscisic acid and other small molecules in Arabidopsis and grasses. Plant Methods https://doi. org/10.1186/s13007-019-0423-y (2019).

60. Nour-Eldin, H. H., Hansen, B. G., Norholm, M. H. H., Jensen, J. K. \& Halkier, B. A. Advancing uracil-excision based cloning towards an ideal technique for cloning PCR fragments. Nucleic Acids Res. https://doi.org/10.1093/nar/gkl635 (2006).

61. Wang, C., Zhang, J. \& Schroeder, J. I. Two-electrode Voltage-clamp Recordings in Xenopus laevis Oocytes: Reconstitution of Abscisic Acid Activation of SLAC1 Anion Channel via PYL9 ABA Receptor. Bio-protocol https://doi.org/10.21769/BioProtoc.2114 (2017).

62. Wang, $\mathrm{C}$. et al. Reconstitution of $\mathrm{CO} 2$ regulation of SLAC1 anion channel and function of CO2-permeable PIP2;1 aquaporin as CARBONIC ANHYDRASE4 interactor. Plant Cell 28, 568-582 (2016).

63. Wu, F. H. et al. Tape-Arabidopsis Sandwich-a simpler Arabidopsis protoplast isolation method. Plant Methods 5, 16 (2009).

64. Gao, X., Chen, J., Dai, X., Zhang, D. \& Zhao, Y. An effective strategy for reliably isolating heritable and Cas9-Free Arabidopsis mutants generated by CRISPR/Cas9-mediated genome editing. Plant Physiol. 171, 1794-1800 (2016)

65. Gao, Y. et al. Auxin binding protein 1 (ABP1) is not required for either auxin signaling or Arabidopsis development. Proc. Natl Acad. Sci. USA 112, 2275-2280 (2015)

66. Gao, Y. \& Zhao, Y. Self-processing of ribozyme-flanked RNAs into guide RNAs in vitro and in vivo for CRISPR-mediated genome editing. J. Integr. plant Biol. 56, 343-349 (2014).

\section{Acknowledgements}

This research was funded by National Institutes of Health (GM060396-ES010337) and National Science Foundation (MCB-1900567) grants to J.I.S., a Postdoctral Fellowship for Research Abroad from the Japan Society for the promotion of Science to Y.T., a Ciencias sem Fronteiras/CNPq fellowship (203406/2014-1) to P.H.O.C. and a scholarship from the China Scholarship Council to L.Z., an EMBO long-term post-doctoral fellowship (ALTF334-2018) to G.D., and in part NIH grant GM114660 to Y.Z. We thank Dr. Majid Ghassemian (University of California, San Diego) for advice at analyzing LC-MS MS data (National Institutes of Health grant number: S10OD021724). We thank Mr. 
Brian Chang and Ms. Katie H. Lee (University of California, San Diego) for help with amiRNA lines. We thank Ms. Krystal Bosmans and Mr. Thien Trac (University of California, San Diego) for help with producing recombinant proteins.

\section{Author contributions}

Y.T. and J.I.S. conceived of the project; Y.T., J.Z, P.H., F.H., and J.I.S. designed research; Y.T., J.Z., P.H., P.H.O.C., L.Z., G.D., S.M., C.G., and F.H. performed experiments; C.G. and Y.Z. generated CRISPR/Cas9 plants; Y.T., J.Z., P.H., P.H.O.C., L.Z., G.D., S.M., C.G., Y.Z., F.H., and J.I.S. analyzed data; and Y.T., J.Z., P.H., F.H., and J.I.S. wrote the manuscript.

\section{Competing interests}

The Authors declare the following competing interests: The University of California San Diego has submitted a patent (US patent $2 / 816,492$, pending) on behalf of Y.T., J.I.S., and F.H. on M3K-dependent modulation of abscisic acid signaling and osmotic stress-linked responses. The other authors declare no competing interests.

\section{Additional information}

Supplementary information is available for this paper at https://doi.org/10.1038/s41467019-13875-y.

Correspondence and requests for materials should be addressed to J.I.S.
Peer review information Nature Communications thanks the anonymous reviewers for their contribution to the peer review of this work. Peer reviewer reports are available.

Reprints and permission information is available at http://www.nature.com/reprints

Publisher's note Springer Nature remains neutral with regard to jurisdictional claims in published maps and institutional affiliations.

(c) (i)

Open Access This article is licensed under a Creative Common Attribution 4.0 International License, which permits use, sharing, adaptation, distribution and reproduction in any medium or format, as long as you give appropriate credit to the original author(s) and the source, provide a link to the Creative Commons license, and indicate if changes were made. The images or other third party material in this article are included in the article's Creative Commons license, unless indicated otherwise in a credit line to the material. If material is not included in the article's Creative Commons license and your intended use is not permitted by statutory regulation or exceeds the permitted use, you will need to obtain permission directly from the copyright holder. To view a copy of this license, visit http://creativecommons.org/ licenses/by/4.0/.

(c) The Author(s) 2020 